\title{
Influence of probe geometry on measurement results of non-ideal thermal conductivity sensors
}

\author{
Patrick Tiefenbacher, Norbert I. Kömle, Wolfgang Macher, and Günter Kargl \\ Space Research Institute, Austrian Academy of Sciences, Graz, Austria \\ Correspondence to: Patrick Tiefenbacher (patrick.tiefenbacher@oeaw.ac.at)
}

Received: 19 November 2015 - Published in Geosci. Instrum. Method. Data Syst. Discuss.: 10 February 2016 Revised: 13 July 2016 - Accepted: 1 August 2016 - Published: 6 September 2016

\begin{abstract}
The thermal properties of the surface and subsurface layers of planets and planetary objects yield important information that allows us to better understand the thermal evolution of the body itself and its interactions with the environment. Various planetary bodies of our Solar System are covered by so-called regolith, a granular and porous material. On such planetary bodies the dominant heat transfer mechanism is heat conduction via IR radiation and contact points between particles. In this case the energy balance is mainly controlled by the effective thermal conductivity of the top surface layers, which can be directly measured by thermal conductivity probes. A traditionally used method for measuring the thermal conductivity of solid materials is the needleprobe method. Such probes consist of thin steel needles with an embedded heating wire and temperature sensors. For the evaluation of the thermal conductivity of a specific material the temperature change with time is determined by heating a resistance wire with a well-defined electrical current flowing through it and simultaneously measuring the temperature increase inside the probe over a certain time. For thin needle probes with a large length-to-diameter ratio it is mathematically easy to derive the thermal conductivity, while this is not so straightforward for more rugged probes with a larger diameter and thus a smaller length-to-diameter ratio. Due to the geometry of the standard thin needle probes they are mechanically weak and subject to bending when driven into a soil. Therefore, using them for planetary missions can be problematic.

In this paper the thermal conductivity values determined by measurements with two non-ideal, ruggedized thermal conductivity sensors, which only differ in length, are compared to each other. Since the theory describing the temperature response of non-ideal sensors is highly complicated,
\end{abstract}

those sensors were calibrated with an ideal reference sensor in various solid and granular materials. The calibration procedure and the results are described in this work.

\section{Introduction}

One way for a better understanding of our Solar System is a better knowledge of the objects in it, the planets and their satellites, as well as asteroids and comet nuclei. An important part of this knowledge is the physical properties of their surface layers, i.e. mechanical, optical, electrical and thermal properties. The last one is the focus of this work. Thermal conductivity of the materials comprising the solid near-surface layers of the planetary bodies mentioned above is of high interest for the following reasons. One reason is that for modelling the thermal evolution of any planetary body the average heat flux across the surface is an important boundary condition (Hofmeister et al., 2007). Another reason for the importance of measuring these thermal properties is that they control, together with the other physical properties mentioned above, the processes on a planetary surface to a great extent (Kömle, 2005). Moreover, variations in thermal conductivity can be an indicator of the presence of water-saturated soil or ice, because dry soil has a significantly smaller thermal conductivity than wet soil or ice (Incropera et al., 2007).

However, measurements of thermal properties on planetary bodies, except on the Earth, are still scarce. To date thermal properties have only been measured in situ for two terrestrial bodies - the Moon and Mars. Apollo 15 (1971) and Apollo 17 (1972) carried the Apollo Lunar Surface Experimental Package (ALSEP) for measuring the thermal con- 
ductivity and the vertical temperature gradient on the Moon (Langseth et al., 1972, 1973). The first in situ measurements of thermal properties on Mars were carried out by the Thermal and Electrical Conductivity Probe (TECP) on board the Phoenix lander in 2008 (Zent et al., 2010). The latest instrument designed for in situ measurements of the surface and subsurface temperatures of comet 67P/ChuryumovGerasimenko is MUPUS - Multipurpose Sensors for Surface and Sub-Surface Science - a part of the scientific payload of the Philae lander. MUPUS is composed of three separate instruments: (i) a cylinder-shaped thermal probe, MUPUSPEN, designed to be inserted into the cometary surface by a hammer mechanism; (ii) an infrared radiometer, MUPUSTM; and (iii) a thermal sensor and accelerometer in each of the two harpoon anchors of the lander. MUPUS started to operate in November 2014, but since the harpoon anchors failed to fire and since MUPUS-PEN could not be fully hammered into the (hard) soil, subsurface thermal measurements could not be performed (Spohn et al., 2015). There is thus a need for further geophysical in situ exploration of the surface and subsurface of moons, comets and planets in future lander missions. This is particularly necessary to allow us more detailed insights into the composition and evolution of those planetary bodies, especially for prospecting water ice and water deposits or other resources. For example, such instruments are recommended for the payload of the upcoming Russian Lunar Polar Lander missions, which have the goal to investigate the properties of the regolith in the lunar south polar regions by in situ measurements and by returning samples to Earth (ESA, 2014). These areas are of particular interest, because they are expected to contain water ice at shallow depths.

In the middle of the last century one can find the first theoretical descriptions and experiments concerning so-called single probes. They are described among others by Blackwell (1952) and Blackwell (1954). For the single-probe method, a single-needle probe is used, which at the same time serves as a heater and as a temperature sensor. The basic principle of this method is to heat the cylindrical probe embedded in the respective sample material by a constant power supply for a defined time interval, while the temperature response is measured simultaneously. The thermal properties of the surrounding medium influence the temperature increase in a way that allows their determination from the measured temperature response.

For the thermal conductivity measurements we make use of various needle sensors built by Dutch company Hukseflux. Model TP02 - Thermal Probe 02 (Hukseflux, 2010) - serves as the standard reference sensor. It is used to calibrate the custom-made models, denoted as LNP03, Lunar Probe 03, and LNP04, Lunar Probe 04, which are mechanically more robust. Thick probes are preferable over thin needles when applied to granular materials of high porosity. Therefore they are better suited to be used as a payload for planetary surface missions than standard probes. The geometric dimensions of these customized sensors lie between the TECP, with a needle length of $1.5 \mathrm{~cm}$, and MUPUS-PEN, which has a length of $35 \mathrm{~cm}$. This work lays the basis for further investigations into the effects of probe geometry. The motivation is to make a significant contribution towards the development of a reliable and accurate thermal properties instrument for future planetary lander missions. We focus on the properties of the sensors and methods and the accuracy of data evaluation, but do not treat other aspects of the development of a spacequalified instrument.

In the following sections one can find the theoretical approach for needle-shaped thermal sensors, a short presentation of the used measurement probes and sample materials, the results of thermal conductivity measurements in various sample materials, as well as the calibration of the LNP sensors and a comparison between LNP03 and LNP04.

\section{Theoretical background and data evaluation technique}

Generally there are two main groups of thermal conductivity measurement methods: on the one hand, the steady-state methods, where a constant temperature difference is established over time. For these methods a complex and expensive measurement system is needed. Therefore these methods are not optimal for field and space applications.

On the other hand the non-steady-state or transient methods, where usually a needle-shaped heater embedded in a sample is heated over a certain time interval, and the temperature response, which depends on the thermal properties of the sample, is measured at or near the heater at the same time. Due to the smaller complexity and costs of these systems they are more suitable for field and space applications. For measurements done for this work a transient method using needle-shaped heater/sensor combinations with a different geometry was chosen. The theory of this method is outlined below.

\subsection{Transient thermal conductivity probes}

According to Carslaw and Jaeger (1959) an infinite line heat source placed in an infinite, homogeneous and isotropic medium is the general approach for the line heat source technique. As described in Özisik (1989) the heat conduction equation of such a cylinder-symmetric, one-dimensional boundary value problem can be written as

$$
\frac{\partial^{2} T}{\partial r^{2}}+\frac{1}{r} \frac{\partial T}{\partial r}+\frac{Q(r, t)}{k}=\frac{1}{\kappa} \frac{\partial T}{\partial t} ; \quad 0 \leq r<\infty, t>0,
$$

where $T$ is the temperature (K), $t$ the characteristic time (s), $r$ is the radial distance from the centre $(\mathrm{m}), Q(r, t)$ denotes a volume heat source $\left(\mathrm{Wm}^{-3}\right), k$ is the thermal conductivity $\left(\mathrm{Wm}^{-1} \mathrm{~K}^{-1}\right)$ and $\kappa$ the thermal diffusivity $\left(\mathrm{m}^{2} \mathrm{~s}^{-1}\right)$ of the ambient medium. The following initial and boundary conditions are to be satisfied: 


$$
T=T_{0} \text { for } \begin{array}{r}
0 \leq r<\infty, t=0 . \\
r \rightarrow \infty, t>0 .
\end{array}
$$

A general solution can be obtained by integral transform, in this case a Hankel transform (Özisik, 1989):

$$
\begin{aligned}
& T(r, t)=T_{0}+\int_{\beta=0}^{\infty} \beta J_{0}(\beta r) \exp \left(-\kappa \beta^{2} t\right) \\
& \cdot\left[\frac{\kappa}{k} \int_{t^{\prime}=0}^{t} \exp \left(\kappa \beta^{2} t^{\prime}\right) \mathrm{d} t^{\prime} \int_{r^{\prime}=0}^{\infty} r^{\prime} J_{0}\left(\beta r^{\prime}\right) Q\left(r^{\prime}, t^{\prime}\right) \mathrm{d} r^{\prime}\right] \mathrm{d} \beta,
\end{aligned}
$$

where $T_{0}$ is the ambient temperature $(\mathrm{K})$ and $J_{0}$ is the Bessel function of the first kind and of order zero.

\subsubsection{Infinite line heat source with neglected sensor properties}

For many applications it is sufficient to consider an infinite line heat source $Q_{1}\left(\mathrm{Wm}^{-1}\right)$ immersed in an infinite medium positioned at $r=0$. Being aware that in practice the heat source can not be a perfect line and that neither the heat source nor the surrounding medium is infinite, various errors arise from this idealization. Nevertheless, this approach allows a good first estimate of the thermal conductivity of the respective medium, as will be described below. For an infinite line heat source $Q_{1}$ positioned in the centre $(r=0)$ that supplies energy at a constant rate, the volume power density $Q(r, t)\left(\mathrm{Wm}^{-3}\right)$ can be defined as

$Q(r, t)=\frac{Q_{1}}{2 \pi r} \delta(r-0)$,

where $\delta(r-0)$ is the Dirac delta function at $r=0$. By substituting Eq. (3) into Eq. (2) an expression for the temperature response to the heat emitted from the line heat source is obtained:

$T(r, t)=T_{0}+\frac{1}{4 \pi k} \int_{t^{\prime}=0}^{t} \frac{Q_{1}(t)}{\left(t-t^{\prime}\right)} \exp \left(\frac{-r^{2}}{4 \kappa\left(t-t^{\prime}\right)}\right) \mathrm{d} t^{\prime}$,

and, for $Q_{1}=$ const.,

$$
\begin{aligned}
& T(r, t)=T_{0}+\frac{Q_{1}}{4 \pi k} \int_{\frac{r^{2}}{4 \kappa t}}^{\infty} \frac{\exp (-u)}{u} \mathrm{~d} u \\
& =T_{0}+\frac{Q_{1}}{4 \pi k} E_{1}\left(\frac{r^{2}}{4 \kappa t}\right)
\end{aligned}
$$

where $E_{1}(x)=\int_{x}^{\infty} \frac{\exp (-u)}{u} \mathrm{~d} u$ is denoted as an exponential integral function, which is defined as a particular definite integral of the ratio between an exponential function and its argument. As described in Abramowitz and Stegun (1964), this integral can also be expressed by a series expansion:

$$
\begin{aligned}
E_{1}(x) & =-\gamma-\ln x-\sum_{n=1}^{\infty} \frac{(-1)^{n} x^{n}}{n n !} \\
& =-\gamma-\ln x+x-\frac{1}{4} x^{2}+\mathcal{O}\left(x^{3}\right) .
\end{aligned}
$$

The constant $\gamma=0.5772 \ldots$ is known as the EulerMascheroni constant or Euler's constant. The contributions in Eq. (6) after the logarithmic term can be neglected for small $x$ values (equivalent to large $t$ values). Thus, using Eq. (5) and Eq. (6), the temperature change can be approximated by

$T(r, t)-T_{0}=\frac{Q_{1}}{4 \pi k}\left[-\gamma+\ln t+\ln \left(\frac{4 \kappa}{r^{2}}\right)+\ldots\right]$.

Furthermore, the variation of temperature with the natural logarithm of time as the independent variable can be written as

$\frac{\mathrm{d} T}{\mathrm{~d} \ln t}=\frac{Q_{1}}{4 \pi k}$.

This formula shows that, after an initial non-linear temperature rise, a linear relationship between the probe temperature and the natural logarithm of time is established. With knowledge of the heating power and the temperature increase as a function of time, the thermal conductivity can be derived from Eq. (8):

$k=\frac{Q_{1}}{4 \pi}\left(\frac{\mathrm{d} T}{\mathrm{~d} \ln t}\right)^{-1}$.

An improvement over this idealized geometry is the determination of a heat source of finite radius in a similar analytical way as described below. Moreover, in Hütter and Kämle (2012) it is explained that the evaluation approach for a line heat source can also be used for large hollow cylindrical geometries if measurement time is long enough.

\subsubsection{Heat source with thermal surface resistance and non-ideal sensor properties}

The theory presented in the previous section is idealized in various ways. It is assumed that the heat source is concentrated in a line and that there is negligible sensor heat capacity and thermal conductance across the probe-sample interface. However, this is not the case for real probes. Every real sensor has finite dimensions and characteristic thermal properties differing from the sample properties. Furthermore, there exists a more or less high thermal contact resistance at the boundary between sensor and sample material, depending on the composition and structure of the sample.

Solutions of the heat equation for this problem have been developed amongst others by Jaeger (1956). These re- 
lations include a non-negligible heat capacity of the sensor $S\left(\mathrm{Jm}^{-1} \mathrm{~K}^{-1}\right)$ and a finite thermal conductance $H^{1}$ $\left(\mathrm{Wm}^{-2} \mathrm{~K}^{-1}\right) . S$ is the specific volumetric heat capacity of the sensor material per sensor length multiplied by the sensor cross section:

$S=c_{\text {sen }} \rho_{\text {sen }} A_{\text {sen }}$.

The general solution, Eq. (14), can be derived by introducing three dimensionless variables, where all the basic parameters of the problem are included:

$\tau=\frac{\kappa t}{r_{\mathrm{sen}}^{2}}$,

$\alpha=\frac{2 \pi r_{\mathrm{sen}}^{2} \rho c_{p}}{S}$

$h=\frac{k}{r_{\mathrm{sen}} H}$.

$\tau$ is called the Fourier number, and it represents the characteristic time for the propagation of the heat wave originating from a heated cylinder with radius $r_{\text {sen }}(\mathrm{m})$ in the surrounding medium.

$\alpha$ represents the ratio of the sample heat capacity to that of the sensor, where $\rho$ is the density $\left(\mathrm{kg} \mathrm{m}^{-3}\right)$ and $c_{p}$ the specific heat capacity $\left(\mathrm{Jkg}^{-1} \mathrm{~K}^{-1}\right)$ of the sample material.

$h$ is proportional to the ratio of the sample conductivity to the thermal surface conductance between sensor and surrounding sample.

Assuming that the thermal conductivity of the sensor probe is large compared to that of the sample material, that the cylinder is in thermal equilibrium with the surrounding medium at $t=0(T(r, 0)=0)$ and that heat is supplied at the rate per unit length $Q_{1}$ for a defined time interval to the cylinder core; the temperature $T$ in the core at time $t$ is given by the following formulae (Jaeger, 1956):

$T=G(h, \alpha, \tau)=\frac{2 Q_{1} \alpha^{2}}{k \pi^{3}} \int_{0}^{\infty} \frac{\left[1-\exp \left(-\tau u^{2}\right)\right]}{u^{3} f(u)} d u$

with

$$
\begin{aligned}
f(u) & =\left[u J_{0}(u)-\left(\alpha-h u^{2}\right) J_{1}(u)\right]^{2} \\
& +\left[u Y_{0}(u)-\left(\alpha-h u^{2}\right) Y_{1}(u)\right]^{2} .
\end{aligned}
$$

$J_{0}$ and $J_{1}$ are the regular Bessel functions of the first kind and of order zero and one, respectively; $Y_{0}$ and $Y_{1}$ are the Bessel functions of the second kind and of order zero and one. For small values of $\tau$ the so-called short time approximation is obtained:

$T_{\text {short }-t}=\frac{Q_{1} \alpha}{2 \pi k}\left[\tau-\frac{\alpha \tau^{2}}{2 h}+\mathcal{O}\left(\tau^{\frac{5}{2}}\right)\right]$, if $h \neq 0$,

\footnotetext{
${ }^{1}$ Thermal resistance is the inverse of thermal conductance $H$, i.e. $H \rightarrow \infty$ is equivalent to $(1 / H) \rightarrow 0$ (there is no temperature discontinuity across the respective boundary) (Incropera et al., 2007).
}

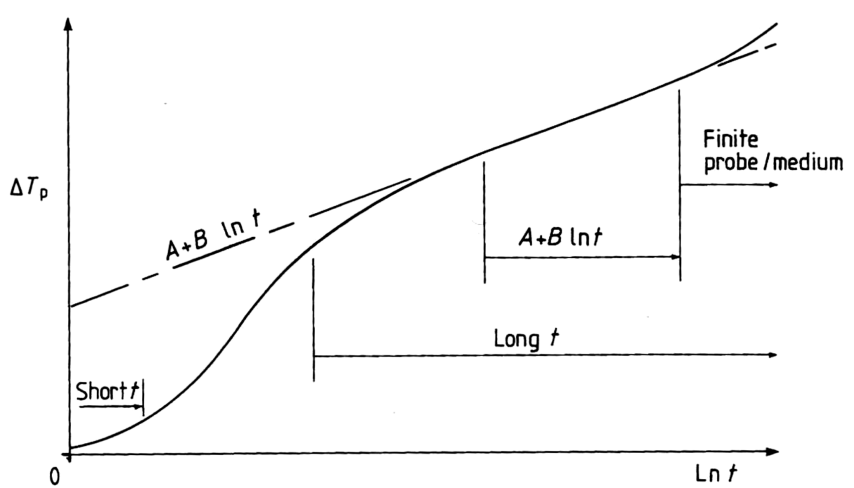

Figure 1. Qualitative heating curve measured with a line heat sensor (Jones, 1988).

or

$T_{\text {short }-t}=\frac{Q_{1} \alpha}{2 \pi k}\left[\tau-\frac{4 \alpha}{3 \pi^{\frac{1}{2}}} \tau^{\frac{3}{2}}+\mathcal{O}\left(\tau^{2}\right)\right]$, if $h=0$.

For large values of $\tau$ the long time approximation is obtained:

$$
\begin{aligned}
T_{\text {long }-t} & =\frac{Q_{1}}{4 \pi k}\left[2 h+\ln \left(\frac{4 \tau}{C}\right)-\frac{(4 h-\alpha)}{2 \alpha \tau}\right. \\
& \left.+\frac{(\alpha-2)}{2 \alpha \tau} \ln \left(\frac{4 \tau}{C}\right)+\ldots\right],
\end{aligned}
$$

where $C=\exp (\gamma)=1.7811 \ldots$ with Euler's constant $\gamma=$ $0.5772 \ldots$ Equation (17) becomes Eq. (7) (when the initial temperature is set to zero) for the case of a very thin sensor and vanishing thermal contact resistance between sensor probe and sample material. It follows from Eq. (17) that a plot of $T$ against $\ln t$ has a linear asymptote of slope $Q_{1} /(4 \pi k)$, and so the thermal conductivity of the sample material $k$ can be determined immediately if $Q_{1}$ is known.

Furthermore, in Macher et al. (2013) another interesting case for the development of thermal conductivity sensors is studied, which will not be discussed here. They considered temperature evolution in and around a heated infinite cylinder with a tubular sheath, assuming non-negligible surface resistances between core and sheath of the cylinder and between the sheath and the surrounding medium.

\subsection{Data evaluation}

The characteristic temperature response of a linear heat source ( $T$ vs. $\ln t)$ to heating is shown in Fig. 1 after Jones (1988).

Four main segments can be identified in this curve. The initial phase after the onset of heating can be used for the evaluation of the thermal conductivity of the sample by the so-called short time approximation (Eq. 16a). It is followed by the non-linear second segment, where self-heating of the probe and contact resistance between probe and sample material are dominant, and thus this approximation is no longer 
valid. The end of this segment is defined by a transition to the linear portion where $T$ depends linearly on $\ln t$. With respect to time this is the section between transient time and maximal measurement time defined in Sect. 2.2.1. This part of the curve is used for the evaluation of the thermal conductivity of the sample by linear regression. Jones (1988) and others used a non-linear long time approximation for the heating curve. They considered a probe with finite diameter and nonnegligible contact resistance between probe and sample material. The non-linear equation is equivalent to Eq. (17) and reads as follows:

$T=A+B \ln t+C \frac{\ln t}{t}+D \frac{1}{t}+\mathcal{O}\left(\frac{\ln t}{t^{2}}\right)$,

with the coefficients

$$
\begin{aligned}
& A=\frac{Q_{1}}{4 \pi k}\left[\ln \left(\frac{4 \kappa}{r_{\mathrm{sen}}^{2}}\right)-\gamma+\frac{2 k}{r_{\mathrm{sen}} H}\right]=\frac{Q}{4 \pi k} X \\
& B=\frac{Q_{1}}{4 \pi k} \\
& C=\frac{Q_{1}}{4 \pi k} \frac{r_{\mathrm{sen}}^{2}}{2 \kappa}\left(1-\frac{\kappa S}{\pi r_{\mathrm{sen}}^{2} k}\right), \\
& D=\frac{Q_{1}}{4 \pi k} \frac{r_{\mathrm{sen}}^{2}}{2 \kappa}\left[\ln \left(\frac{4 \kappa}{r_{\mathrm{sen}}^{2}}\right)+(1-\gamma)-\frac{\kappa S}{\pi r_{\mathrm{sen}}^{2} k} X\right]
\end{aligned}
$$

The second term of Eq. (18) with the coefficient $B$, given in Relation (19b), is equivalent to Eq. (8). From this formula the thermal conductivity $k$ can be easily determined. The other terms of the equation with their respective coefficients can be used to evaluate the other unknown parameters of the sensor probe and the sample. These are the thermal diffusivity $\kappa$ of the sample, the surface conductance $H$ between probe and sample, and the heat capacity per unit length $S$ of the sensor. The last segment of the curve, beginning at the maximum measurement time, is non-linear again, due to finite sample dimensions and axial errors. Hütter (2011) describes two different behaviours of the curve in this last section, providing information about the measurement system. An increasing curve can be observed if the heat wave reaches the wall of the sample container and its conductivity is lower than that of the sample. A decrease in the curve in this section can be observed if convection starts to play a role or if the conductivity of the sample container wall is higher than that of the sample.

Temperature-time data, the heating current and the heater resistance are necessary input data for the evaluation of thermal conductivity. This information, together with the estimated transient and maximum measurement times, was integrated into an evaluation program written in MATLAB.

\subsubsection{Time period for evaluation}

Due to the non-ideal measurement conditions, there are some limitations for the evaluation that have to be considered. The thermal diffusivity $\kappa$ of the sample to be measured and the geometric dimensions of the respective sample and sensor are the key parameters. Two characteristic times can be defined and should be estimated before the measurement.

The transient time $t_{\text {trans }}$ is the time from the onset of heating $(t=0)$ until the beginning of the linear part of the $T-\ln t$ curve (see Fig. 1). This time can be estimated according to Goodhew and Griffiths (2004) as

$t_{\text {trans }}=\frac{50 r_{\mathrm{sen}}^{2}}{4 \kappa}$,

where $r_{\text {sen }}$ is the radius of the sensor probe. This formula is valid for a full cylinder with radius $r_{\text {sen }}$.

The maximum measurement time $t_{\max }$ is the other characteristic time. It is caused by the finite dimension of the sample containers in laboratory measurements. The reason for the existence of this upper time limit is that the heat wave originating from the needle probe reaches the boundary of the sample container after some time, depending on the thermal properties of the respective sample and the distance of the sensor from the boundary. The boundary condition stated in Sect. 2.1, $T=T_{0}$ as $r \rightarrow \infty$, is violated when the thermal wave reaches the boundary of the sample container. Therefore one must always make sure that the duration of one measurement does not exceed $t_{\max }$. Once the heat wave is reflected from the boundaries, the temperature profile is influenced in a way not included in the theory. According to Goodhew and Griffiths (2004), the maximum measurement time can be estimated by the formula

$t_{\max }=\frac{0.6\left(r_{\mathrm{sample}}-r_{\mathrm{sen}}\right)^{2}}{4 \kappa}$,

where $r_{\text {sample }}(\mathrm{m})$ is the radius of the sample container.

\subsubsection{Error assessment}

There are numerous error sources which are responsible for the uncertainty of the thermal conductivity determined by the various sensors. For the estimation of an overall error, the laws of error propagation were used. Generally the resulting quantity $y$ of an experiment is a function of several measured variables $x_{i}$ :

$y=f\left(x_{i}\right)$.

Variations of the variables $x_{i}$ cause the error $\mathrm{d} y$ of $y$, which can be expressed as

$\mathrm{d} y=\sum \frac{\partial y}{\partial x_{i}} \mathrm{~d} x_{i}$

The focus of this work is the evaluation of the thermal conductivity. The function $f\left(x_{i}\right)$ is the relation used for this calculation, defined as

$k(Q, s)=\frac{Q}{4 \pi s}$. 
The parameter $s$ denotes the slope of the linear part of the measurement curve when the measured temperature $T$ is plotted against $\ln t$, which is used for the evaluation. Equation (24) can be rewritten in terms of the measurement parameters:

$k=\frac{R I_{\text {heat }}^{2}}{4 \pi} \frac{\Delta \ln t}{\Delta T}$,

where $R$ is the electrical resistance per unit length $\left(\Omega \mathrm{m}^{-1}\right)$ of the heating wire of the respective sensor needle and $I_{\text {heat }}$ is the applied constant heating current (A). Based on Relation (23) the variation of the thermal conductivity $k$ due to variations of the measured quantities can be written as

$\delta k=\frac{\partial k}{\partial R} \delta R+\frac{\partial k}{\partial I_{\text {heat }}} \delta I_{\text {heat }}+\left(\delta \frac{\mathrm{d} \ln t}{\mathrm{~d} T}\right)\left(\frac{R I^{2}}{4 \pi}\right)$.

Evaluation of Eq. (26) according to Relation (23) leads to the relative error for the thermal conductivity measurement:

$\frac{\delta k}{k}=\frac{\delta R}{R}+2 \frac{\delta I_{\text {heat }}}{I_{\text {heat }}}-\frac{\delta\left(\frac{\mathrm{d} T}{\mathrm{~d} \ln t}\right)}{\frac{\mathrm{d} T}{\mathrm{~d} \ln t}}$.

The form $\frac{\mathrm{d} T}{\mathrm{~d} \ln t}$ describes the slope of the $T-\ln t$ curve from linear regression and is denoted in the following by

$\mu=\frac{\mathrm{d} T}{\mathrm{~d} \ln t}$.

With this definition the last term of Eq. (27) can be approximated as

$-\frac{\delta \mu}{\mu}=\left(\frac{\delta t_{2}}{t_{2}}-\frac{\delta t_{1}}{t_{1}}\right) \frac{1}{\ln t_{2}-\ln t_{1}}-\frac{\delta T_{2}-\delta T_{1}}{T_{2}-T_{1}}$.

The subscripts behind the parameters $T$ and $t$ describe points on the $T-\ln t$ curve, where 1 corresponds to the point at the beginning of the linear part of the curve and 2 corresponds to the end of the linear part (compare Fig. 1). The following two assumptions can be made.

First, the error of the measurement time can be neglected:

$\delta t_{1} \approx \delta t_{2} \approx 0$

Second, the temperature error for the temperature values at the beginning of the linear part of the $T$ - $\ln t$ curve is assumed to be approximately the same as for the values at the end of the linear part of the curve, and is defined as

$\left|\delta T_{1}\right| \approx\left|\delta T_{2}\right| \equiv \delta T$.

So Eq. (29) can be rewritten as

$$
\frac{|\delta \mu|}{\mu}=2 \frac{\delta T}{T_{2}-T_{1}}
$$

With this simplification, and since all the measurement errors are independent of each other, Eq. (27) yields

$\frac{|\delta k|}{k}=\sqrt{\left(\frac{\delta R}{R}\right)^{2}+\left(2 \frac{\delta I}{I}\right)^{2}+\left(2 \frac{\delta T}{T_{2}-T_{1}}\right)^{2}}$.

The third term in this formula shows the relative temperature error, which is the ratio of the error of the temperature measurement to the temperature increase in the linear regime. It can be seen that the relative error is linked to the slope of the measurement curve: the bigger the temperature difference $\left(T_{2}-T_{1}\right)$, the steeper the slope and the smaller the relative temperature error. The gradient of the increase in the slope depends on the sample material.

If linear regression is applied, the following form can be used for the evaluation of the relative error of thermal conductivity:

$\frac{|\delta k|}{k}=\sqrt{\left(\frac{\delta R}{R}\right)^{2}+\left(2 \frac{\delta I}{I}\right)^{2}+\left(\frac{\delta \mu}{\mu}\right)^{2}}$,

where the resulting slope and its error from the regression (of the $T-\ln t$ curve) are substituted for $\mu$ and $\delta \mu$. The variation of the thermal conductivity is caused by the variations of the heater resistance $R$, the heating current $I_{\text {heat }}$ and the uncertainties of the measured temperature $T$ (or of the slope $\mu$ from regression), as given in Eqs. (33) and (34). Generally all these variations consist of uncertainties from needle probe fabrication, used temperature sensors and uncertainties from power supplies and data acquisition units. The maximum possible deviation of a parameter is evaluated by summing up all the variations influencing the measurement. In Eq. (34) for error propagation $\delta R, \delta I$ and $\delta \mu$ are the result of bias (systematic errors) as well as measurement errors, as indicated in the following, where the most important error sources are described.

Resistance error $(\delta R)$ : the resistance of the heating wire cannot be measured continuously, so the measurement precision of a typical digital multimeter used for measuring the resistance is taken into account (see Table 1). The heating wires embedded in all sensors are made of the alloy constantan, which has a constant resistance of $49 \mu \Omega$ at temperatures between 20 and $500{ }^{\circ} \mathrm{C}$ (Heusler, 2014). Therefore the bias caused by the change in resistance with temperature is negligible.

Heating current error $(\delta I)$ : the constant current flowing through the heating wire is generated by the TTi power supply and continuously measured by the Agilent data logger. Both devices are a source of measurement error. The first term in the $\frac{\delta I}{I}$ column in Table 1 is the measurement error caused by the data logger according to Agilent (2012) and the second term the measurement error caused by the power supply according to TTi (2003). These errors are determined for each measurement.

Time error $(\delta t)$ : the time reading error was assumed to be negligible. 
Table 1. Error estimates of the quantities measured during thermal conductivity measurement, listed for the different sensors.

\begin{tabular}{lccc}
\hline Sensor & $\frac{\delta R}{R}$ & $\frac{\delta I}{I}$ & $\frac{\delta T}{T} *$ \\
\hline \multirow{2}{*}{ TP02 } & $\frac{ \pm(0.0005 \cdot R+0.1 \Omega)}{R}$ & $\frac{ \pm\left(0.0005 \cdot I+5 \times 10^{-6} \mathrm{~A}\right) \pm(0.002 \cdot I+0.005 \mathrm{~A})}{I}$ & $\frac{ \pm(0.0001 \cdot R+0.01 \Omega)}{R}$ \\
LNP03 & $\frac{ \pm(0.0005 \cdot R+0.1 \Omega)}{R}$ & $\frac{ \pm(0.001 \cdot I+0.0001 \mathrm{~A}) \pm(0.002 \cdot I+0.005 \mathrm{~A})}{I}$ & $\frac{ \pm(0.0001 \cdot R+0.01 \Omega)}{R}$ \\
LNP04 & $\frac{ \pm(0.0005 \cdot R+0.1 \Omega)}{R}$ & $\frac{ \pm(0.001 \cdot I+0.0001 \mathrm{~A}) \pm(0.002 \cdot I+0.005 \mathrm{~A})}{I}$ & $\frac{ \pm(0.0001 \cdot R+0.01 \Omega)}{R}$ \\
\hline
\end{tabular}

* The relative error of the temperature measurement was calculated as the relative error of the resistance measurement as given in the Agilent data sheet (Agilent, 2012).

Temperature error $(\delta T)$ : the temperature at the TP02 sensor is determined by a differential thermocouple measurement. The reference temperature above the sample is measured by a Pt1000 resistance temperature detector (RTD), mounted in the base of the sensor, for establishing the absolute medium temperature. Due to the differential measurement of the two thermocouples, there is no additional bias and the error is essentially due to the RTD resistance measurement. The Pt1000 RTD is also used in the LNP03 and LNP04 sensors. RTDs are sensors for measuring temperature in virtue of the correlation of the RTD resistance with temperature. So the relative error of the temperature measurement is of the same order of magnitude as the relative error of the measured resistance. The errors caused by the Agilent data logger (Agilent, 2012) are taken into account (see Table 1), while the bias of the RTD can be neglected due to its small tolerance range at the given temperature.

Finally, the scattering (variance) of the data points in the $T-\ln t$ curve contributes to the error $\frac{\delta \mu}{\mu}$ in Eq. (34). However, this contribution is much smaller than the error estimation $\frac{\delta T}{T}$ given in Table 1 . Therefore Eq. (33) is used instead of Eq. (34), providing a good estimate for the total relative error of each determined conductivity value (as indicated by error bars in Figs. 8 to 13). In each figure the standard deviation $\sigma$, belonging to the respective measurement sequences, are given, calculated by

$\sigma=\sqrt{\sum_{i=1}^{n} \frac{\left(x_{i}-\bar{x}\right)^{2}}{n-1}}$,

where $n$ is the number of conductivity values $x_{i}$ per sequence and $\bar{x}$ is their mean value. In fact, the variance of the conductivity values (as plotted in these figures) is well represented by the estimated error bars (apart from some outliers which are to be expected from a statistical point of view).

\section{Applied probes, samples and measurement procedures}

\subsection{Measurement probes}

The thermal conductivity of the various samples was measured with different measurement probes. On the one hand a commercial thermal conductivity sensor, denoted as TP02, and on the other hand two different versions of custom-made sensors, LNP03 and LNP04, all manufactured by Dutch company Hukseflux, were used. The acronyms have been defined by Hukseflux, where TP stands for Thermal Probe and LNP means Lunar Probe. The LNP sensors were designed to investigate their suitability for space application. They are shorter and thicker and therefore more rugged than the commercial TP02 sensor, which can be easily deformed by bending when used in granular material with larger grain sizes or in a material with internal cohesion. However, this type of sensor is planned to be used in regolith but not icy regolith, as it can be assumed that for the deployment in icy regolith the sensors have to be much more robust, according to the findings of Spohn et al. (2015). Due to the smaller length-todiameter ratio of the LNP probes, the evaluation of thermal conductivity is not as straightforward as it is for the "optimal" line heat sources described in the previous section. Additional calibration is necessary. The LNP03 and LNP04 sensors are constructed identically, except for their length. The LNP04 sensor is $20 \mathrm{~mm}$ shorter and thus a little more robust than the LNP03, but the length-to-diameter ratio is smaller. In the following the individual probes are described in more detail.

\subsubsection{TP02 - Thermal Probe 02}

The TP02 sensor is a commercial non-steady-state probe (NSSP) for thermal conductivity measurement. The NSSP measurement method is also known as the transient line heat source, thermal needle, hot needle, heat pulse and hot wire technique described in Sect. 2. Generally such a probe consists of a heating wire, representing a perfect line heat source, and a temperature sensor capable of measuring the temperature at the heat source region. For investigation of a medium, the probe is inserted into it. Measurements with the TP02 sensor are absolute, i.e. there is in principle no need for reference materials.

Three TP02 sensors were used for our measurements, which only differ in heater resistance. The sensor has a diameter of $1.5 \mathrm{~mm}$ and a length of $150 \mathrm{~mm}$, where the uppermost $100 \mathrm{~mm}$ are actively heated by an embedded constantan heating wire. This implies a length-to-diameter ratio of 


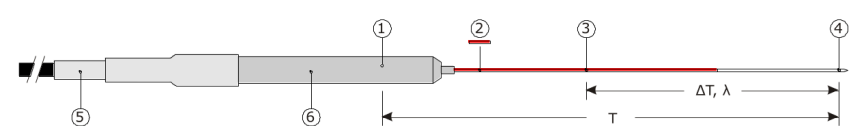

Figure 2. The TP02 probe consists of a needle with two thermocouple junctions; the hot joint (3), the cold joint (4) (remains at a stable temperature) and a heating wire (2). In base (6) a reference temperature sensor (Pt1000) (1) is mounted (Hukseflux, 2010).

the heated segment of 66. According to Kämle et al. (2013), sensors with a ratio of 60 or more fulfil the requirements for the evaluation based on line heat source theory, so the TP02 sensor meets this requirement. All described dimensions can be found in Fig. 3. For temperature measurement the sensor incorporates two thermocouple junctions (thermocouple type $\mathrm{K}$ ) producing a voltage output that is proportional to $\Delta T$ between the heated and unheated parts. One thermocouple is positioned $50 \mathrm{~mm}$ from the base in the heated part, the other one in the unheated tip. Additionally a Pt1000 RTD in the base measures the surrounding temperature and enables the evaluation of the absolute medium temperature. The structure of the TP02 is shown in Figs. 2 and 3. This design provides optimal accuracy independent of the medium temperature and a minimal sensitivity to thermal gradients. The TP02 sensor was used as a reference sensor for calibration of the custom-made LNP03 (Kämle et al., 2013) and LNP04 sensors, as described in Sect. 5.

For the evaluation of the thermal conductivity of a sample measured with the TP02 probe, the thermocouple readings have to be converted from voltage into temperature. Therefore, as described in Hukseflux (2010), the temperaturedependent sensitivity of the thermocouple $E_{\text {sen }}\left(\mathrm{VK}^{-1}\right)$ is calculated as

$E_{\text {sen }}=10^{-6}\left(a_{0}+a_{1} T-a_{2} T^{2}\right)$,

with the constants $a_{0}=39.40 \mathrm{VK}^{-1}, a_{1}=0.050 \mathrm{VK}^{-2}$ and $a_{2}=0.0003 \mathrm{VK}^{-3}$.

The Pt1000 RTD gives the temperature of the sensor's surroundings. So with its readings the absolute temperature $T$ can be calculated. Temperature change measured close to the heating wire is then derived as

$\Delta T=\frac{U_{\text {sen }}}{E_{\mathrm{sen}}}$,

where $U_{\text {sen }}$ is the thermoelectric voltage generated at the thermocouple hot junction. According to Hukseflux (2010) the sensitivity given in Eq. (36) should be accurate within $1 \%$ in the temperature range from -40 to $100{ }^{\circ} \mathrm{C}$.

With the Pt1000, RTD resistance values are measured, which have to be converted into temperature values. The relation between resistance $R$ and temperature $T$ of platinum RTDs is described by the Callendar-van Dusen equation (McGee, 1988):

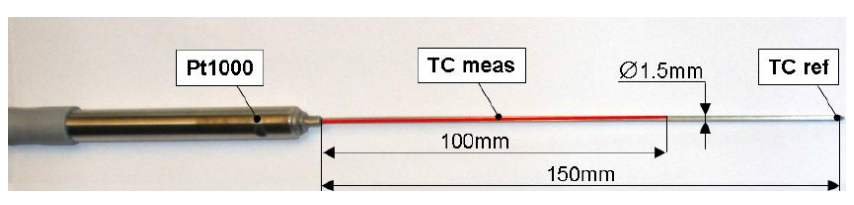

Figure 3. Commercial TP02 thermal conductivity probe (Hütter, 2011).

$$
\begin{aligned}
& R=R_{0}\left[1+A T+B T^{2}+C T^{3}(T-100)\right], \\
& R=R_{0}\left[1+A T+B T^{2}\right],
\end{aligned}
$$

where Eq. (38a) is valid for the temperature range of -200 to $0^{\circ} \mathrm{C}$ and Eq. (38b) for the temperature range of 0 to $850^{\circ} \mathrm{C}$. The constants have the following values:

$$
\begin{aligned}
& A=+3.9083 \times 10^{-3}{ }^{\circ} \mathrm{C}^{-1}, \\
& B=-5.7750 \times 10^{-7}{ }^{\circ} \mathrm{C}^{-2}, \\
& C=-4.1830 \times 10^{-12 \circ} \mathrm{C}^{-3} .
\end{aligned}
$$

These equations and the values of the constants are especially valid for platinum RTDs, so no further calibration is necessary. If the RTD element is made from another material, the constants $A, B$ and $C$ have to be determined separately (Schaffer, 2014).

\subsubsection{LNP03 - Lunar Probe 03}

The LNP03 sensor is a custom-made prototype thermal conductivity sensor. There is one sensor, the LNP-A (further denoted as LNP03-needle 4), which has a mounting stud with a screw thread at the top for installing it on a deployment device (for example, a robotic arm on a planetary lander). In relation to the whole needle such a part has a relatively large mass and heat capacity and could therefore influence the measurements. Thus, two identical sensors, the LNP-B (further denoted as LNP03-needle 2 and LNP03-needle 3), were built consisting only of the needle and the necessary heating and measurement parts without such a mounting stud, but with the same geometric dimensions. Both versions are shown in Fig. 4.

The sensor has a needle length of $100 \mathrm{~mm}$ and a diameter of $3.5 \mathrm{~mm}$, which implies a length-to-diameter ratio of 28 . This is far below the minimal ratio of 60 for evaluation on the basis of the line heat source theory, but the sensor is more robust than the TP02 sensor and can hardly be bent. For the heating of the LNP03 sensor a constantan wire is embedded in the needle, which extends over the whole needle length. The temperature response to heating is measured with three Pt1000 RTDs placed at the following positions (see Fig. 4): close to the tip, in the centre of the needle and close to the base of the needle. The conversion of the resistance values measured by the RTDs to temperature values is described in Sect. 3.1.1. Only the values of the middle sensor were used 


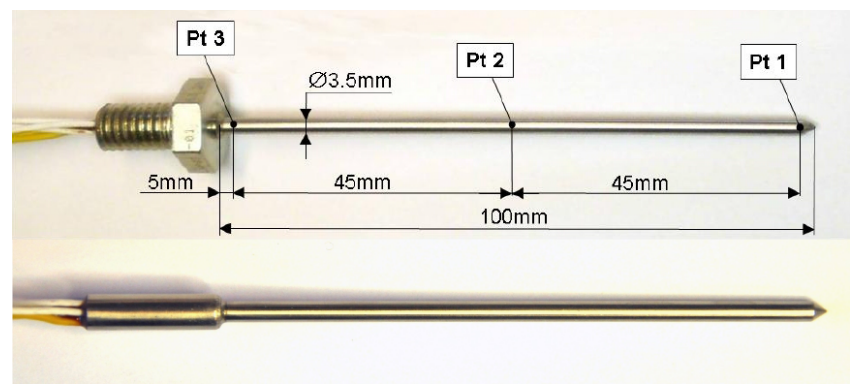

Figure 4. LNP03 thermal conductivity probe prototype. The upper picture shows the LNP-A, further denoted as LNP03-needle 4, and the lower picture shows the LNP-B, which is further denoted as LNP03-needle 2 and LNP03-needle 3.

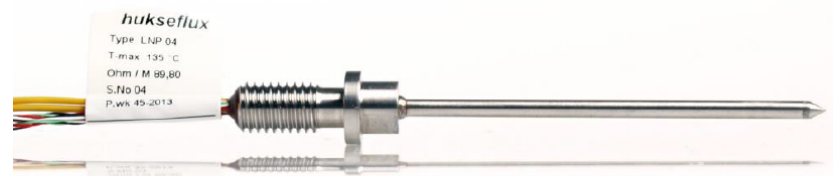

Figure 5. LNP04 thermal conductivity probe prototype (Hukseflux, 2013).

for the evaluation of the thermal conductivity. The reason is that it is supposed that the RTD close to the needle base is significantly influenced by the heat capacity of the mounting stud, while the influence of the finite length of the needle on the RTD at the tip is not really understood yet. The temperature is measured with a four-wire technique to increase the measurement accuracy.

\subsubsection{LNP04 - Lunar Probe 04}

The LNP04 sensor is the further developed version of the LNP03 custom-made prototype and is shown in Fig. 5. The differences are the needle length and the construction of the mounting stud. There are four identical sensor needles in use. As the LNP-A, the LNP04 sensor is equipped with a mounting stud with a screw thread on the top for installing it onto a deployment device, but this one has less mass and thus less heat capacity which could influence the measurements.

With a length of $80 \mathrm{~mm}$ the LNP04 sensor is $20 \mathrm{~mm}$ shorter than the LNP03 but has the same diameter, implying a length-to-diameter ratio of 22. This is also far below the minimal ratio of 60 for evaluation on the basis of the line heat source theory, but the sensor is even more robust than the LNP03 sensor. The heating and measurement properties are the same as for the LNP03 sensor. For the heating a constantan wire is embedded in the needle, which extends over the whole needle length. The temperature response to heating is measured with three Pt1000 RTDs placed at the following

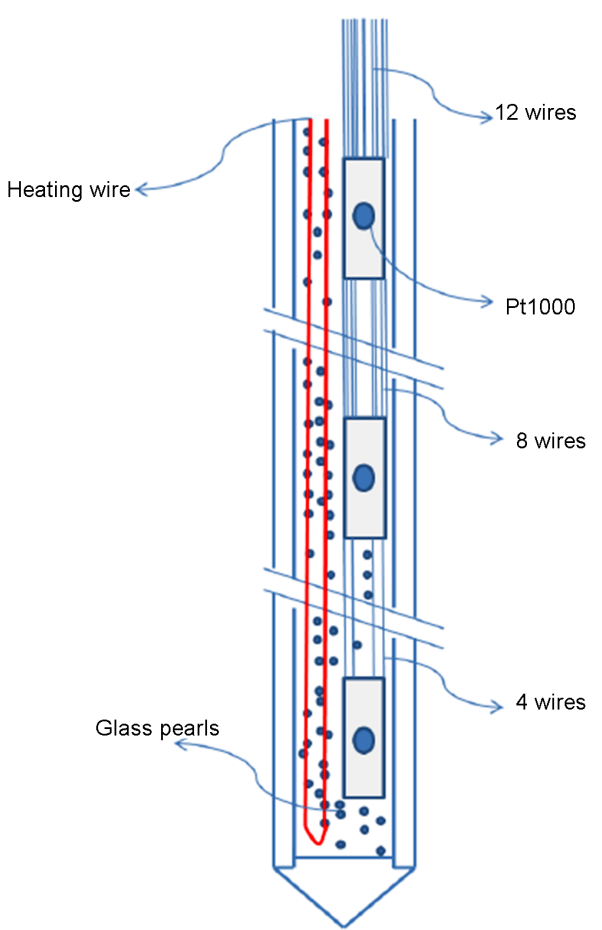

Figure 6. Sketch of the LNP04 sensor, showing its internal construction (Hukseflux, 2013).

positions (see Fig. 6): close to the tip, in the centre of the needle, and close to the base of the needle. The conversion of the resistance values measured by the RTDs to temperature values is described in Sect. 3.1.1. As for LNP03, only the values of the middle sensor were used for evaluation and the temperature was measured with a four-wire technique to increase measurement accuracy.

\subsection{Sample materials}

The conductivities of a variety of samples were investigated. The choice of the sample materials, which are summarized in Table 2, was primarily motivated by the requirement of wellknown thermal material properties. Another aspect taken into account was the high porosity of the media, as can be found in planetary regolith. Therefore we used a granular material, glass beads, in three different size fractions. The determination of the physical bulk properties of granular material, as needed for the glass bead samples, is described in Appendix A.

\subsection{Measurement procedure}

For the analysis, the thermal model explained in Sect. 2.1.2, considering a heat source with thermal surface resistance and non-ideal properties, was used. The thermal conductivity was evaluated using the following standard procedure, as illustrated in Fig. 7. 
Table 2. Overview of the sample materials.

\begin{tabular}{|c|c|c|}
\hline Agar & $\begin{array}{l}\text { Physical properties: (water at } 20^{\circ} \mathrm{C} \text { ) } \\
\text { Thermal conductivity } k \text { : } \\
\text { Heat capacity } c_{p}: \\
\text { Density } \rho \text { : } \\
\text { Dimensions: } 30 \times 30 \mathrm{~cm}(\mathrm{D} \times \mathrm{H}) \\
\text { Medium: agar-water mixture }\end{array}$ & $\begin{array}{l}0.60 \mathrm{Wm}^{-1} \mathrm{~K}^{-1} \\
4.18 \mathrm{~kJ} \mathrm{~kg}^{-1} \mathrm{~K}^{-1} \\
998.21 \mathrm{~kg} \mathrm{~m}^{-3}\end{array}$ \\
\hline Glass beads & $\begin{array}{l}\text { Physical properties: } \\
\text { Thermal conductivity } k \text { : } \\
\text { Heat capacity } c_{\mathrm{p}} \text { : } \\
\text { Bulk density } \rho_{\text {bulk: }}: \\
\text { Dimensions: } 30 \times 40 \mathrm{~cm}(\mathrm{D} \times \mathrm{H}) \\
\text { Medium: soda lime glass }\end{array}$ & $\begin{array}{l}0.19 \mathrm{Wm}^{-1} \mathrm{~K}^{-1} \\
1.201 \mathrm{~kJ} \mathrm{~kg}^{-1} \mathrm{~K}^{-1} \\
1510 \mathrm{~kg} \mathrm{~m}^{-3}\end{array}$ \\
\hline $1.00-1.25 \mathrm{~mm}$ & $\begin{array}{l}\text { Physical properties: } \\
\text { Thermal conductivity } k \text { : } \\
\text { Heat capacity } c_{p} \text { : } \\
\text { Bulk density } \rho_{\text {bulk }} \text { : } \\
\text { Dimensions: } 35 \times 28 \times 30 \mathrm{~cm}(\mathrm{~L} \times \mathrm{H} \times \mathrm{W}) \\
\text { Medium: soda lime glas }\end{array}$ & $\begin{array}{l}0.19 \mathrm{Wm}^{-1} \mathrm{~K}^{-1} \\
1.195 \mathrm{~kJ} \mathrm{~kg}^{-1} \mathrm{~K}^{-1} \\
1470 \mathrm{~kg} \mathrm{~m}^{-3}\end{array}$ \\
\hline $3.80-4.30 \mathrm{~mm}$ & $\begin{array}{l}\text { Physical properties: } \\
\text { Thermal conductivity } k \text { : } \\
\text { Heat capacity } c_{p}: \\
\text { Bulk density } \rho_{b u l k}: \\
\text { Dimensions: } 26 \times 28 \mathrm{~cm}(\mathrm{D} \times \mathrm{H}) \\
\text { Medium: soda lime glass }\end{array}$ & $\begin{array}{l}0.19 \mathrm{Wm}^{-1} \mathrm{~K}^{-1} \\
1.193 \mathrm{~kJ} \mathrm{~kg}^{-1} \mathrm{~K}^{-1} \\
1450 \mathrm{~kg} \mathrm{~m}^{-3}\end{array}$ \\
\hline Kerafol KP96 & $\begin{array}{l}\text { Physical properties: } \\
\text { Thermal conductivity } k \text { : } \\
\text { Density } \rho \text { : } \\
\text { Dimensions: } 16 \times 17 \mathrm{~cm}(\mathrm{D} \times \mathrm{H}) \\
\text { Medium: thermally conductive grease }\end{array}$ & $\begin{array}{l}2.40 \mathrm{Wm}^{-1} \mathrm{~K}^{-1} \\
2600 \mathrm{~kg} \mathrm{~m}^{-3}\end{array}$ \\
\hline PE & $\begin{array}{l}\text { Physical properties: } \\
\text { Thermal conductivity } k \text { : } \\
\text { Heat capacity } c_{p} \text { : } \\
\text { Density } \rho \text { : } \\
\text { Dimensions: } 30 \times 18.5 \mathrm{~cm}(\mathrm{D} \times \mathrm{H}) \\
\text { Medium: high-density polyethylene }\end{array}$ & $\begin{array}{l}0.41 \mathrm{Wm}^{-1} \mathrm{~K}^{-1} \\
1.70-2.40 \mathrm{~kJ} \mathrm{~kg}^{-1} \mathrm{~K}^{-1} \\
950 \mathrm{~kg} \mathrm{~m}^{-3}\end{array}$ \\
\hline Water ice & $\begin{array}{l}\left.\text { Physical properties: (water ice at }-20^{\circ} \mathrm{C}\right) \\
\text { Thermal conductivity } k \text { : } \\
\text { Heat capacity } c_{p} \text { : } \\
\text { Density } \rho \text { : } \\
\text { Dimensions: } 26 \times 25 \mathrm{~cm}(\mathrm{D} \times \mathrm{H}) \\
\text { Medium: water ice }\end{array}$ & $\begin{array}{l}2.33 \mathrm{Wm}^{-1} \mathrm{~K}^{-1} \\
1.94 \mathrm{kJkg}^{-1} \mathrm{~K}^{-1} \\
919.40 \mathrm{~kg} \mathrm{~m}^{-3}\end{array}$ \\
\hline
\end{tabular}

1. Removal of any temperature trend from the data not associated with the active heating of the sensor.

2. Identification of the suitable interval of the measured temperature profile (linear part on the $T-\ln t$ graph).

3. Calculation of the thermal conductivity using the method of linear regression as described in Sect. 2.2.
The commercial TP02 sensor as well as prototypes LNP03 and LNP04 were tested by conducting measurements in different materials of well-known thermal conductivity and comparison of the results to tabulated values. All measurements were performed under atmospheric pressure, at room temperature $\left(21-24^{\circ} \mathrm{C}\right)$ and a relative humidity of around 

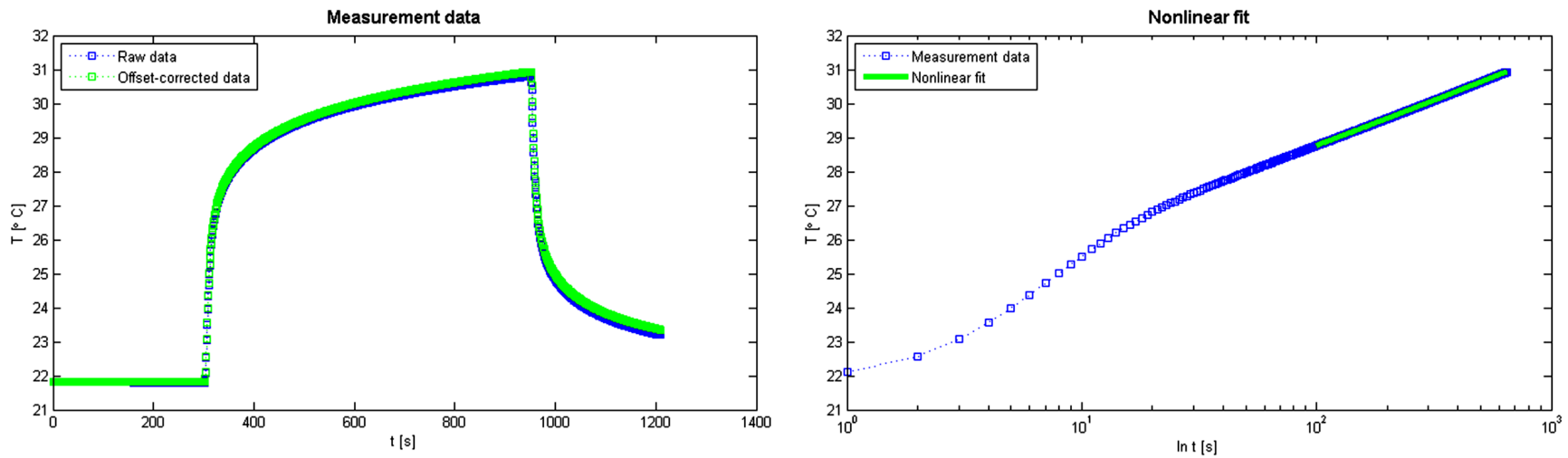

Figure 7. Thermal conductivity evaluation procedure performed in an agar sample with the LNP04 sensor with a heating time of $600 \mathrm{~s}$ and a heating power of $1 \mathrm{~W}$ as an example, evaluated and plotted by the MATLAB software. Left: raw data and offset-corrected data of the total measurement ( 0 to $300 \mathrm{~s}$ : phase before heating, used for the evaluation of the offset; 300 to $900 \mathrm{~s}$ : heating phase, used for the evaluation of the thermal conductivity; 900 to $1200 \mathrm{~s}$ : decline phase, which is not used for any evaluation). Right: semi-logarithmic plot of the heating phase with the interval used for the non-linear fit shown in green.

$40 \%$, except for those in water ice, where the sample was stored in a deep-freezer at $-20^{\circ} \mathrm{C}$.

\section{Thermal conductivity measurements for different sample materials}

In the following the measured thermal conductivity of each sample material is listed, where $k_{\text {avg }}$ is the average value (arithmetic mean), $k_{\text {med }}$ is the median of the measured thermal conductivities, and the minimum and maximum conductivity values are denoted as $k_{\min }$ and $k_{\max }$, respectively. The absolute measurement error $|\delta k|$ is the mean value of the estimated errors of the single measurements, determined according to Eq. (33), while $\sigma$ is the mean value of the standard deviations of the single measurements, calculated by Eq. (35). The values of the used LNP03 and LNP04 needles are listed separately.

\subsection{Agar}

All three sensor types, TP02, LNP03 and LNP04, were used for the measurements in agar. The same sample was used for all sensors. Enough sample material was produced to allow removal of the penetrated layer after a measurement with one sensor and to guarantee an untouched sample surface for the other sensor probes. The disadvantage of this method is that one cannot rule out that invisible small cracks will occur at the sample surface due to the insertion of a sensor. If this happens the contact between sensor and medium will get worse. The better but much more time-consuming method would be to produce a new sample for each probe and to put the probes into the agar before it solidifies.

The thermal conductivities determined by the different sensors are summarized in Table 3 and shown in Fig. 8 vs. the chronologically ordered measurements.
Table 3. Thermal conductivity of agar determined by the TP02, the LNP03 and the LNP04 sensor.

\begin{tabular}{lccccccc}
\hline & & \multicolumn{7}{c}{$\mathrm{Wm}^{-1} \mathrm{~K}^{-1}$} \\
\cline { 3 - 8 } Sensor & No. & $k_{\text {avg }}$ & $k_{\text {med }}$ & $\sigma$ & $|\delta k|$ & $k_{\min }$ & $k_{\max }$ \\
\hline TP02 & 5 & 0.575 & 0.601 & 0.041 & 0.064 & 0.489 & 0.631 \\
\hline LNP03 & & & & & & & \\
Needle 2 & 5 & 0.609 & 0.604 & - & 0.023 & 0.603 & 0.623 \\
Needle 3 & 5 & 0.608 & 0.603 & - & 0.024 & 0.602 & 0.627 \\
Needle 4 & 5 & 0.601 & 0.596 & - & 0.023 & 0.592 & 0.613 \\
All & 15 & 0.609 & 0.604 & 0.010 & 0.023 & 0.592 & 0.627 \\
\hline LNP04 & & & & & & & \\
Needle 1 & 5 & 0.622 & 0.615 & - & 0.024 & 0.611 & 0.636 \\
Needle 2 & 5 & 0.592 & 0.590 & - & 0.023 & 0.587 & 0.604 \\
Needle 3 & 5 & 0.619 & 0.615 & - & 0.024 & 0.609 & 0.632 \\
Needle 4 & 5 & 0.642 & 0.643 & - & 0.024 & 0.626 & 0.653 \\
All & 20 & 0.618 & 0.615 & 0.021 & 0.024 & 0.587 & 0.653 \\
\hline \multicolumn{7}{c}{$k_{\text {tabulated }}=0.60 \pm 10 \% \mathrm{Wm}^{-1} \mathrm{~K}^{-1}$} \\
\end{tabular}

\subsection{Glass beads}

Three different single grain size fractions were investigated. Glass beads of 0.25 to $0.50 \mathrm{~mm}$ grain size were used for measurements with the TP02, LNP03 and LNP04 sensors. Thermal conductivity measurements in the glass beads with a grain size of 1.00 to $1.25 \mathrm{~mm}$ and 3.80 to $4.30 \mathrm{~mm}$ were performed only with the TP02 sensor.

The thermal conductivity values are summarized in Tables 4,5 and 6 for the different sizes of the glass beads, respectively. A comparison of the conductivities determined by all three sensors in the smallest glass beads is shown in Fig. 9. Having a look at the values measured by the TP02 sensor in the different sizes of glass beads (see Fig. 10), an increase in thermal conductivity with increasing grain size can be observed. This was already described by Hütter (2011). The 


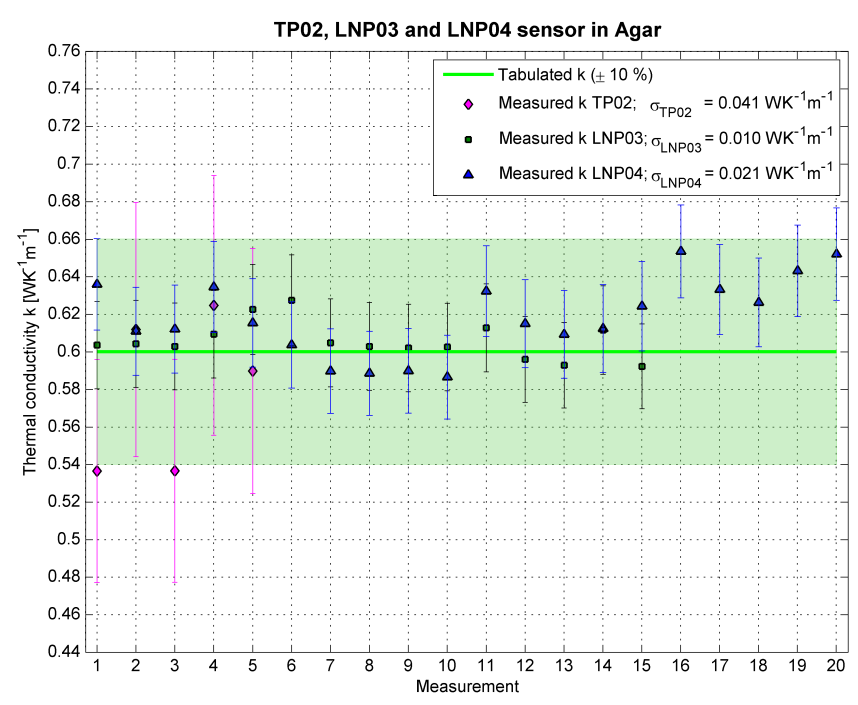

Figure 8. Thermal conductivity values of agar, measured by the TP02, LNP03 and LNP04 sensors. The error bars represent the measurement error as described in Sect. 2.2.2 for each measurement value. The green line indicates the tabulated value of the thermal conductivity (Hukseflux, 2007) with a range of $\pm 10 \%$. In the legend the standard deviation $\sigma$, determined according to Eq. (35), of the respective measurement sequence is given.

Table 4. Thermal conductivity of glass beads with a grain size of 0.25 to $0.50 \mathrm{~mm}$ determined by the TP02, the LNP03 and the LNP04 sensor. The value for $k_{\text {tabulated }}$ is the thermal conductivity of a bulk of glass beads.

\begin{tabular}{lccccccc}
\hline & & \multicolumn{7}{c}{$\mathrm{Wm}^{-1} \mathrm{~K}^{-1}$} \\
\cline { 3 - 8 } Sensor & No. & $k_{\text {avg }}$ & $k_{\text {med }}$ & $\sigma$ & $|\delta k|$ & $k_{\min }$ & $k_{\max }$ \\
\hline TP02 & 10 & 0.164 & 0.163 & 0.012 & 0.018 & 0.141 & 0.188 \\
\hline LNP03 & & & & & & & \\
Needle 2 & 5 & 0.206 & 0.205 & - & 0.008 & 0.205 & 0.209 \\
Needle 3 & 5 & 0.204 & 0.203 & - & 0.008 & 0.200 & 0.207 \\
Needle 4 & 5 & 0.206 & 0.206 & - & 0.008 & 0.206 & 0.208 \\
All & 15 & 0.205 & 0.206 & 0.002 & 0.008 & 0.200 & 0.209 \\
\hline LNP04 & & & & & & & \\
Needle 1 & 5 & 0.203 & 0.203 & - & 0.008 & 0.200 & 0.210 \\
Needle 2 & 5 & 0.206 & 0.205 & - & 0.008 & 0.198 & 0.213 \\
Needle 3 & 5 & 0.213 & 0.213 & - & 0.008 & 0.209 & 0.217 \\
Needle 4 & 5 & 0.211 & 0.208 & - & 0.008 & 0.207 & 0.216 \\
All & 20 & 0.209 & 0.209 & 0.006 & 0.008 & 0.198 & 0.217 \\
\hline \multicolumn{7}{c}{$k_{\text {tabulated }}=0.19 \pm 10 \% \mathrm{Wm}^{-1} \mathrm{~K}^{-1}$} \\
\hline
\end{tabular}

reason for the correlation between bead size and conductivity is that the heat transfer via the gas phase is much more effective than via the contact points of the solid grains. When applied to regoliths under very low pressure or vacuum, grain to grain contacts become increasingly important. Furthermore, it can be said that the precision calculated for glass beads is in principle comparable to that of other granular materials. However, we can expect less accuracy for materials of very
Table 5. Thermal conductivity of glass beads with a grain size of 1.00 to $1.25 \mathrm{~mm}$ determined by the TP02 sensor. The value for $k_{\text {tabulated }}$ is the thermal conductivity of a bulk of glass beads.

\begin{tabular}{lccccccc}
\hline & & \multicolumn{6}{c}{$\mathrm{Wm}^{-1} \mathrm{~K}^{-1}$} \\
\cline { 3 - 8 } Sensor & No. & $k_{\text {avg }}$ & $k_{\text {med }}$ & $\sigma$ & $|\delta k|$ & $k_{\min }$ & $k_{\max }$ \\
\hline TP02 & 5 & 0.176 & 0.180 & 0.012 & 0.019 & 0.159 & 0.192 \\
\hline \multicolumn{6}{c}{$k_{\text {tabulated }}=0.19 \pm 10 \% \mathrm{Wm}^{-1} \mathrm{~K}^{-1}$} \\
\hline
\end{tabular}

Table 6. Thermal conductivity of glass beads with a grain size of 3.80 to $4.30 \mathrm{~mm}$ determined by the TP02 sensor. The value for $k_{\text {tabulated }}$ is the thermal conductivity of a bulk of glass beads.

\begin{tabular}{lccccccc}
\hline & & \multicolumn{6}{c}{$\mathrm{Wm}^{-1} \mathrm{~K}^{-1}$} \\
\cline { 3 - 8 } Sensor & No. & $k_{\text {avg }}$ & $k_{\text {med }}$ & $\sigma$ & $|\delta k|$ & $k_{\min }$ & $k_{\max }$ \\
\hline TP02 & 5 & 0.192 & 0.196 & 0.026 & 0.021 & 0.159 & 0.226 \\
\hline \multicolumn{6}{c}{$k_{\text {tabulated }}=0.19 \pm 10 \% \mathrm{Wm}^{-1} \mathrm{~K}^{-1}$} \\
\hline
\end{tabular}

small conductivity (about $0.01 \mathrm{Wm}^{-1} \mathrm{~K}^{-1}$ or less), because a greater bias is produced due to higher axial heat flow.

\subsection{Kerafol KP96}

Thermal grease Kerafol KP96 was used for measurements with the TP02, LNP03 and LNP04 sensors. It is a viscous fluid, so convection is suppressed, but it is soft enough so that the sensors can be inserted easily. This highly viscous grease was stirred well before conducting the measurements in order to get a homogeneous medium. Despite slow and careful stirring, some air bubbles emerged in the fluid which might affect the measurement of the thermal conductivity. Thus, after stirring the sensor was put into the grease carefully and before starting the measurements we waited some time and slightly tapped against the sample container, so that most of the bubbles were able to rise to the surface and escape.

The thermal conductivities determined by the different sensors are summarized in Table 7 and shown in Fig. 11. In the upper panel it can be seen that the measured thermal conductivity is far below the value given by the manufacturer. Maybe the reason for this high deviation is that the thermal conductivity value given by the manufacturer is only valid when this thermal grease is applied in a thin contact layer and not in the bulk fluid, as used for our measurements. This fact was already observed in the previous measurements described by Kämle et al. (2013).

\subsection{PE - polyethylene}

All three sensors, TP02, LNP03 and LNP04, were used for the measurements in PE. The sensors were inserted into predrilled holes with different diameters and lengths to provide proper fitting. The hole for the TP02 has a diameter of $1.8 \mathrm{~mm}$ and a length of $150 \mathrm{~mm}$, the one for the LNP03 and 
Table 7. Thermal conductivity of Kerafol KP96 determined by the TP02, the LNP03 (needle 2) and the LNP04 sensor.

\begin{tabular}{lccccccc}
\hline & & \multicolumn{7}{c}{$\mathrm{Wm}^{-1} \mathrm{~K}^{-1}$} \\
\cline { 2 - 8 } Sensor & No. & $k_{\text {avg }}$ & $k_{\text {med }}$ & $\sigma$ & $|\delta k|$ & $k_{\min }$ & $k_{\text {max }}$ \\
\hline TP02 & 7 & 0.858 & 0.870 & 0.027 & 0.049 & 0.815 & 0.888 \\
\hline LNP03 & 5 & 0.968 & 0.964 & 0.011 & 0.038 & 0.962 & 0.989 \\
\hline LNP04 & 5 & 0.912 & 0.901 & - & 0.035 & 0.867 & 0.987 \\
Needle 2 & 5 & 0.982 & 0.980 & - & 0.038 & 0.977 & 0.993 \\
Needle 3 & 5 & 0.947 & 0.978 & 0.048 & 0.036 & 0.867 & 0.993 \\
All & 10 & 0.947 & $k_{\text {tabulated }}=2.40 \pm 10 \% \mathrm{Wm}^{-1} \mathrm{~K}^{-1}$ \\
\hline \multicolumn{6}{c}{}
\end{tabular}

Table 8. Thermal conductivity of PE determined by the TP02, the LNP03 and the LNP04 sensor.

\begin{tabular}{lccccccc}
\hline & & \multicolumn{7}{c}{$\mathrm{Wm}^{-1} \mathrm{~K}^{-1}$} \\
\cline { 3 - 8 } Sensor & No. & $k_{\text {avg }}$ & $k_{\text {med }}$ & $\sigma$ & $|\delta k|$ & $k_{\min }$ & $k_{\max }$ \\
\hline TP02 & 5 & 0.429 & 0.425 & 0.043 & 0.044 & 0.370 & 0.478 \\
\hline LNP03 & & & & & & & \\
Needle 2 & 5 & 0.473 & 0.473 & - & 0.015 & 0.469 & 0.479 \\
Needle 3 & 5 & 0.466 & 0.467 & - & 0.014 & 0.464 & 0.467 \\
Needle 4 & 5 & 0.469 & 0.471 & - & 0.015 & 0.459 & 0.477 \\
All & 15 & 0.469 & 0.469 & 0.005 & 0.015 & 0.459 & 0.479 \\
\hline LNP04 & 5 & 0.453 & 0.454 & - & 0.014 & 0.443 & 0.461 \\
Needle 2 & 5 & & & & & & \\
Needle 3 & 5 & 0.467 & 0.467 & - & 0.015 & 0.464 & 0.471 \\
All & 10 & 0.460 & 0.463 & 0.009 & 0.015 & 0.443 & 0.471 \\
\hline \multicolumn{7}{c}{$k_{\text {tabulated }}=0.41 \pm 10 \% \mathrm{Wm}^{-1} \mathrm{~K}^{-1}$} \\
\end{tabular}

LNP04 a diameter of $3.6 \mathrm{~mm}$ and a length of $115 \mathrm{~mm}$. For improvement of the thermal contact between sensor and sample, glycerin was used as a contact fluid. The thermal conductivity of glycerin is around $0.3 \mathrm{Wm}^{-1} \mathrm{~K}^{-1}$ and therefore significantly higher than that of air, which is $0.026 \mathrm{Wm}^{-1} \mathrm{~K}^{-1}$.

The thermal conductivities determined by the different sensors are summarized in Table 8 and shown in Fig. 12.

\subsection{Water ice}

Water ice was used for measurements with the TP02, LNP03 and LNP04 sensors. For that, water was placed in a deepfreezer with the measurement needles inserted and cooled down over several days until a constant temperature of about $-20^{\circ} \mathrm{C}$ was reached. Despite the slight expansion of the sample due to freezing, no visible cracks were formed during the freezing process. This suggests that there was a good contact between sensor and sample material, which is consistent with the low variability of the thermal conductivity values determined by all sensors and the good agreement with the tabulated value. There was no danger of local phase changes in the sample material along the contact surface of the sen-

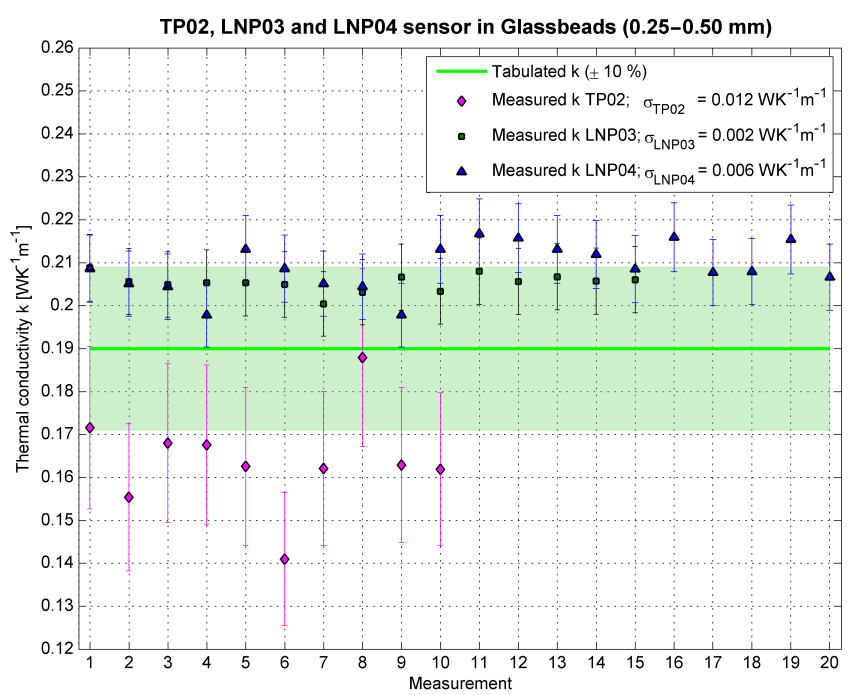

Figure 9. Thermal conductivity values of glass beads with a grain size of 0.25 to $0.50 \mathrm{~mm}$, measured by the TP02, LNP03 and LNP04 sensors. The tabulated value of the thermal conductivity (Hukseflux, 2013) refers to a bulk of glass beads and is indicated by the green line with a range of $\pm 10 \%$. The error bars represent the measurement error as described in Sect. 2.2.2 for each measurement value. In the legend the standard deviation $\sigma$, determined according to Eq. (35), of the respective measurement sequence is given.

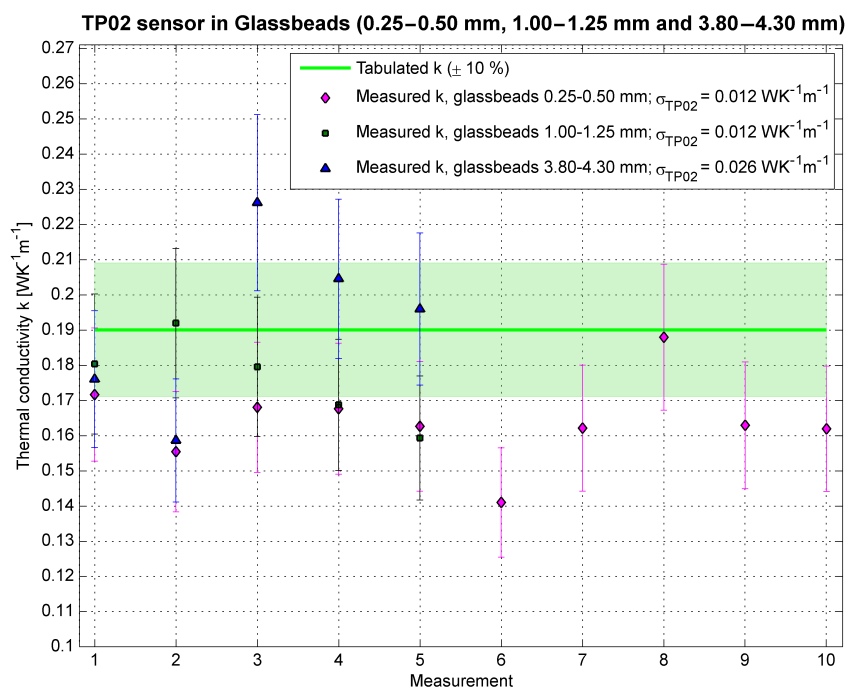

Figure 10. Thermal conductivity values of glass beads in the different size fractions determined by the TP02 sensor. The error bars represent the measurement error as described in Sect. 2.2.2 for each measurement value. The green line indicates the tabulated value of the thermal conductivity (of a bulk of glass beads) (Hukseflux, 2013 ) with a range of $\pm 10 \%$. In the legend the standard deviation $\sigma$, determined according to Eq. (35), of the respective measurement sequence is given. 
Table 9. Thermal conductivity of water ice determined by the TP02, the LNP03 (needle 2) and the LNP04 (needle 2) sensor.

\begin{tabular}{lccccccc}
\hline & & \multicolumn{6}{c}{$\mathrm{Wm}^{-1} \mathrm{~K}^{-1}$} \\
\cline { 3 - 8 } Sensor & No. & $k_{\text {avg }}$ & $k_{\text {med }}$ & $\sigma$ & $|\delta k|$ & $k_{\min }$ & $k_{\max }$ \\
\hline TP02 & 5 & 2.260 & 2.248 & 0.053 & 0.072 & 2.187 & 2.315 \\
LNP03 & 5 & 2.297 & 2.272 & 0.05 & 0.074 & 2.263 & 2.392 \\
LNP04 & 5 & 2.290 & 2.288 & 0.055 & 0.072 & 2.228 & 2.354 \\
\hline \multicolumn{6}{c}{$k_{\text {tabulated }}=2.33 \pm 10 \% \mathrm{Wm}^{-1} \mathrm{~K}^{-1}$} \\
\end{tabular}

Table 10. Thermal conductivity measurement results for the TP02, LNP03, and LNP04 sensors in different sample materials.

\begin{tabular}{lccccc}
\hline \multirow{2}{*}{ Sensor } & \multicolumn{5}{c}{ Thermal conductivity $k\left(\mathrm{Wm}^{-1} \mathrm{~K}^{-1}\right)$} \\
\cline { 2 - 6 } & Glass beads & PE & Agar & KP96 & Water ice \\
\hline TP02 & 0.164 & 0.429 & 0.580 & 0.858 & 2.260 \\
LNP03 & 0.205 & 0.469 & 0.606 & 0.968 & 2.297 \\
LNP04 & 0.209 & 0.460 & 0.618 & 0.947 & 2.290 \\
\hline Tabulated & 0.19 & 0.41 & 0.60 & 2.40 & 2.33 \\
\hline
\end{tabular}

sor and sample, because temperature changes due to sensor heating never exceeded a few degrees.

The thermal conductivities determined by the different sensors are summarized in Table 9 and shown in Fig. 13.

\section{Calibration of the LNP sensors}

The custom-made thermal conductivity sensors LNP03 and LNP04 are rugged prototype sensors. As already mentioned in Kämle et al. (2013), these sensors are necessarily thicker and shorter and so cannot fulfil the requirements for the evaluation of the thermal conductivity by Eq. (8). Thus, there are in principle two possibilities to do this. Either one can use a much more complicated formalism, applying the theory of non-ideal (short and thick) sensors, or one can use the simple theory of ideal sensors with an additional calibration function. While the first method has been described in Hütter (2011), Hütter and Kämle (2012) and Macher et al. (2013), the second method is described in this section. The testing and calibration of the LNP03 sensor were already conducted by Kämle et al. (2013) and is now verified by measurements in partly different sample materials and an adapted measurement set-up. Calibration of the LNP04 sensor was carried out in the same way. As a reference sensor, an off-the-shelf thermal conductivity probe - TP02 - was used. A detailed description of all the sensors was given in Sect. 3.1.
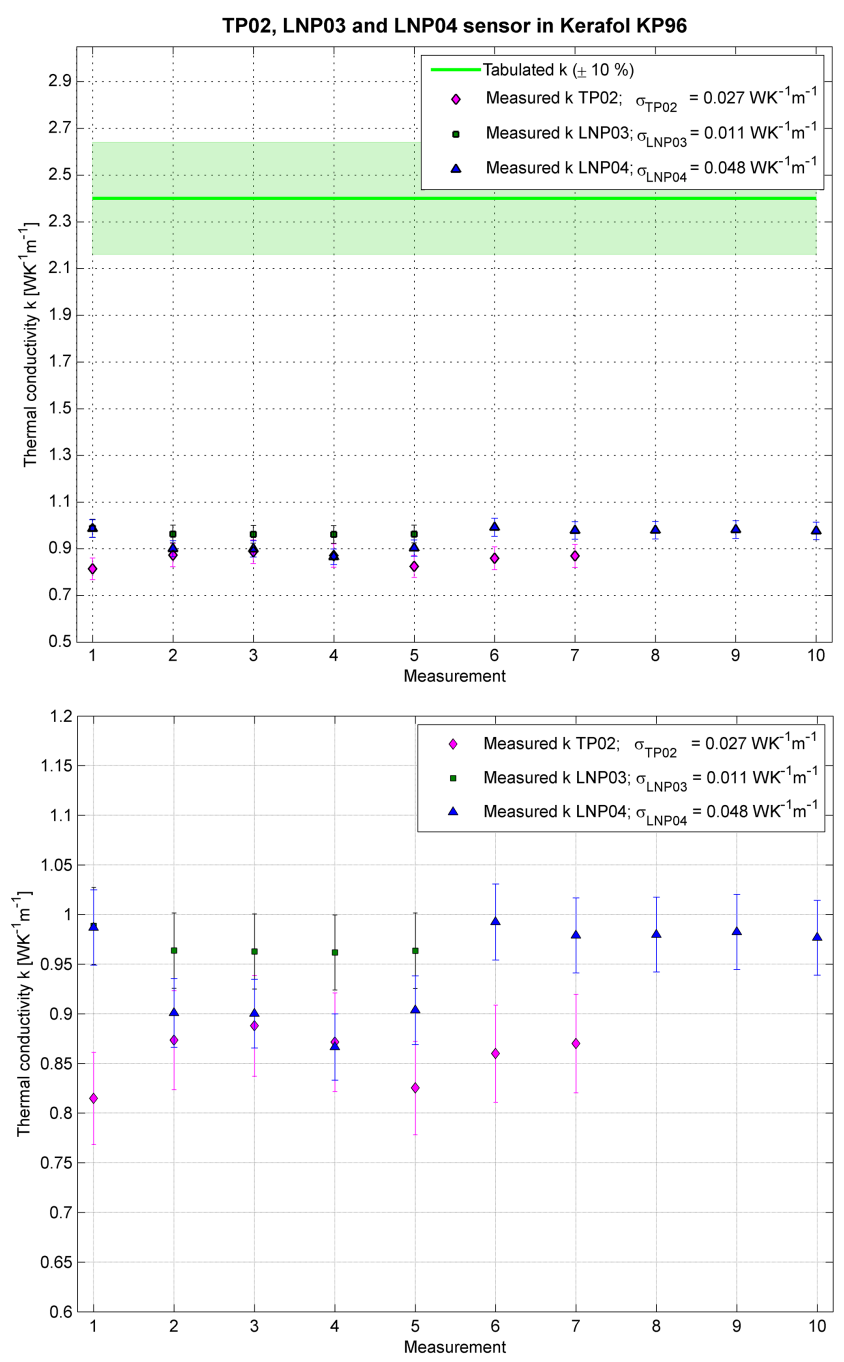

Figure 11. Thermal conductivities of the thermal grease Kerafol KP96 measured by the TP02, LNP03 and LNP04 sensors. In the upper panel, the green line indicates the value of the thermal conductivity given by the manufacturer (Kerafol, 2013) with a range of $\pm 10 \%$. The lower picture shows the range of the measured values in more detail. The error bars represent the measurement error as described in Sect. 2.2.2 for each measurement value. In the legend the standard deviation $\sigma$, determined according to Eq. (35), of the respective measurement sequence is given.

\subsection{Characterization of calibration samples}

For the calibration measurements the used materials were selected in order to cover the range of thermal conductivities from around $0.01 \mathrm{Wm}^{-1} \mathrm{~K}^{-1}$ to around $2 \mathrm{Wm}^{-1} \mathrm{~K}^{-1}$. All measurements for the calibration of the LNP sensors were performed under atmospheric pressure at temperatures in the range of 21 to $24^{\circ} \mathrm{C}$ and a humidity around $40 \%$. The lower end, around $0.10 \mathrm{Wm}^{-1} \mathrm{~K}^{-1}$, corresponds to granular materials. As a representative of such a material, silica glass beads with a grain size in the range of 0.25 to $0.5 \mathrm{~mm}$ were used. 
Table 11. Calibration factors derived for the different materials.

\begin{tabular}{lcccccc}
\hline Sensor & \multicolumn{6}{c}{ Calibration factor (1) } \\
\cline { 2 - 7 } & Glass beads & PE & Agar & KP96 & Water ice & $f_{\text {cal }}$ \\
\hline LNP03-TP02 & 0.799 & 0.914 & 0.957 & 0.886 & 0.984 & 0.908 \\
LNP04-TP02 & 0.786 & 0.932 & 0.938 & 0.906 & 0.987 & 0.909 \\
\hline
\end{tabular}

Table 12. Conversion factors derived for the different materials.

\begin{tabular}{lcccccc}
\hline Sensor & \multicolumn{6}{c}{ Conversion factor (1) } \\
\cline { 2 - 7 } & Glass beads & PE & Agar & KP96 & Water ice & $f_{\text {con }}$ \\
\hline LNP03-LNP04 & 0.983 & 1.020 & 0.980 & 1.022 & 1.003 & 1.002 \\
\hline
\end{tabular}

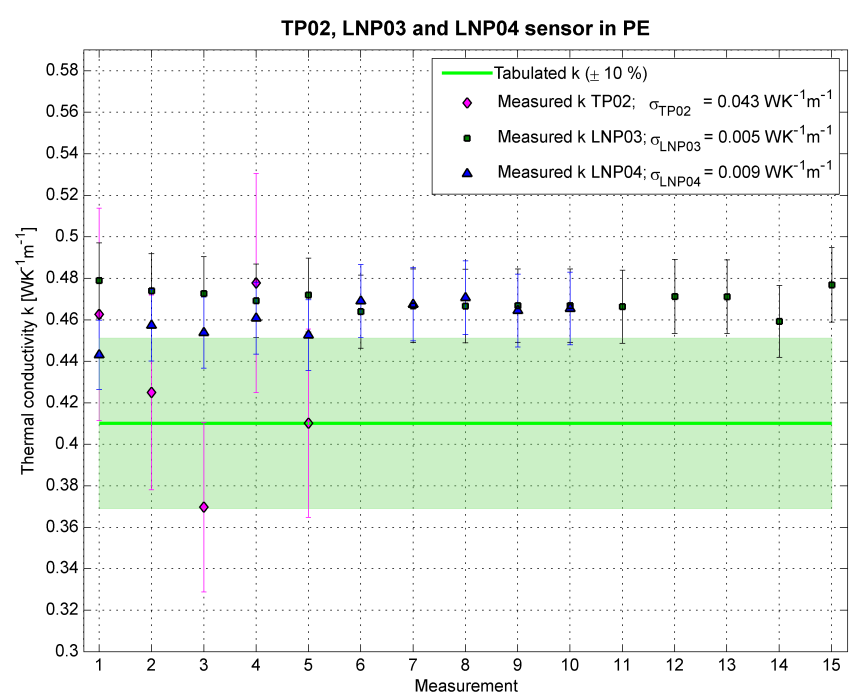

Figure 12. Thermal conductivities of the solid plastic material PE measured by the TP02, LNP03, and LNP04 sensors. The tabulated value of the thermal conductivity (Faigle, 2014) is indicated by the green line with a range of $\pm 10 \%$. The error bars represent the measurement error as described in Sect. 2.2.2 for each measurement value. In the legend the standard deviation $\sigma$, determined according to Eq. (35), of the respective measurement sequence is given.

For a thermal conductivity value of about $0.40 \mathrm{Wm}^{-1} \mathrm{~K}^{-1}$ the solid plastic material $\mathrm{PE}$ and, for the range of 0.50 to $0.60 \mathrm{Wm}^{-1} \mathrm{~K}^{-1}$, water or agar, were used as sample materials. Investigation of the upper end of the range of thermal conductivity we were interested in, around $2 \mathrm{Wm}^{-1} \mathrm{~K}^{-1}$, was performed by using compact water ice. To cover the range around $1 \mathrm{Wm}^{-1} \mathrm{~K}^{-1}$ and to bridge the gap between water (agar) and water ice, the thermally conductive grease Kerafol KP96 was used.

\subsection{Calibration strategy}

For the calibration the following steps were performed.

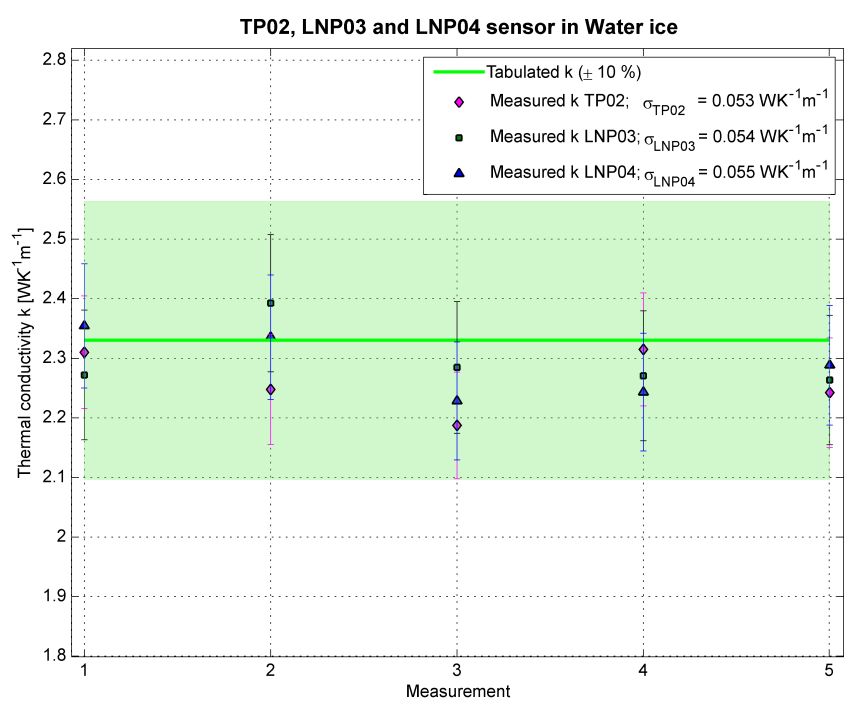

Figure 13. Thermal conductivity values of water ice determined by the TP02, LNP03, and LNP04 sensors. The error bars represent the measurement error as described in Sect. 2.2.2 for each measurement value. The green line indicates the tabulated value of the thermal conductivity of water ice at $-20^{\circ} \mathrm{C}$ (Wikipedia, 2014) with a range of $\pm 10 \%$. In the legend the standard deviation $\sigma$, determined according to Eq. (35), of the respective measurement sequence is given.

1. Reasonably large-sized samples were prepared, which were big enough in diameter and height for the respective sensor. This made sure that no influence from the sample boundaries could disturb the measurements. Estimating the minimum sample sizes can be done by means of the formulae given in Hütter and Kämle (2012).

2. The samples (with sensors inserted) were kept for at least several hours in a thermally stable environment to ensure isothermal conditions for the measurements. All measurements were performed at room temperature, i.e. 


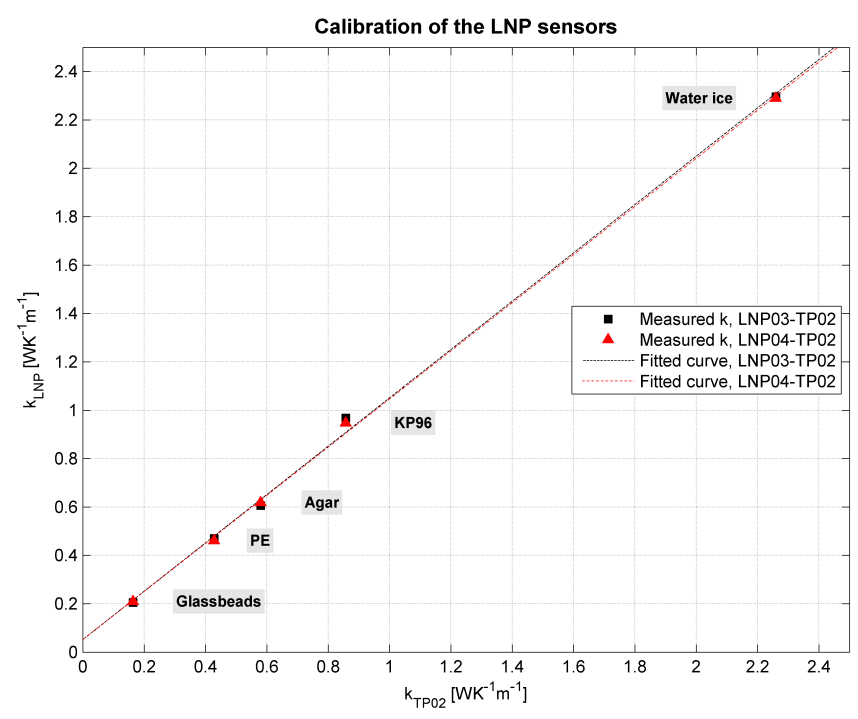

Figure 14. Calibration of the prototypes LNP03 and LNP04 with the commercial TP02 thermal conductivity sensor, which is used as a reference sensor for the calibration measurement. The red symbols represent the mean measurement values of the respective sensor and the dotted line is a linear fit to them.

at an ambient temperature range of 21 to $24^{\circ} \mathrm{C}$, except for those in water ice, where the sample was stored in a deep-freezer at $-20{ }^{\circ} \mathrm{C}$.

3. For the thermal conductivity measurements each sensor was heated separately. To make sure that the sample is in thermal equilibrium again after one measurement procedure, there were long enough time periods (several hours) between two subsequent measurements. The heating periods of the sensors are dependent on the sample and were in the range of 300 to $600 \mathrm{~s}$.

4. Evaluation of the thermal conductivity was performed as described in Sect. 3.3.

The average thermal conductivity results determined from TP02, LNP03 and LNP04 measurements are listed in Table 10 .

\subsection{Calibration results}

The mean value of the individual results from all three sensors was used to evaluate the calibration factors for the different samples, shown in Table 11. The average value calculated from the individual calibration factors is designated as $f_{\text {cal }}$ and given in Table 11 too. So the true thermal conductivity can be obtained from a measurement with the prototype LNP sensor according to the formula

$k_{\mathrm{TP} 02}=f_{\text {cal }} \cdot k_{\mathrm{LNP}}$.

In Fig. 14 the conductivities measured by the LNP sensors vs. that of the TP02 reference sensor can be seen. The symbols represent the mean values listed in Tables 10, while the

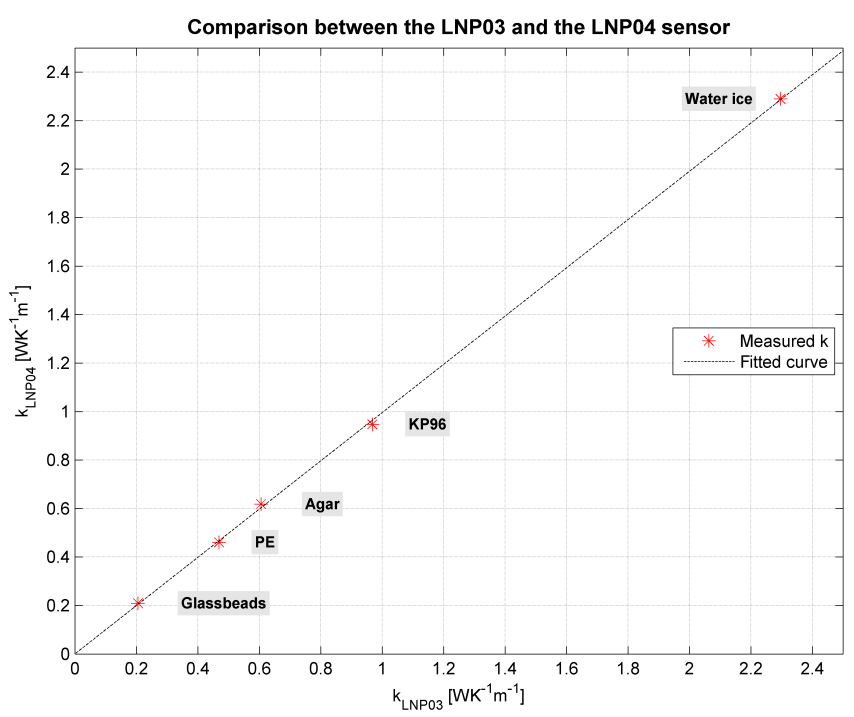

Figure 15. Comparison between the prototype LNP03 and the LNP04 thermal conductivity sensor. The symbols represent the mean measurement values of the LNP03 and the LNP04 sensor, while the dotted line is a linear fit through the data points.

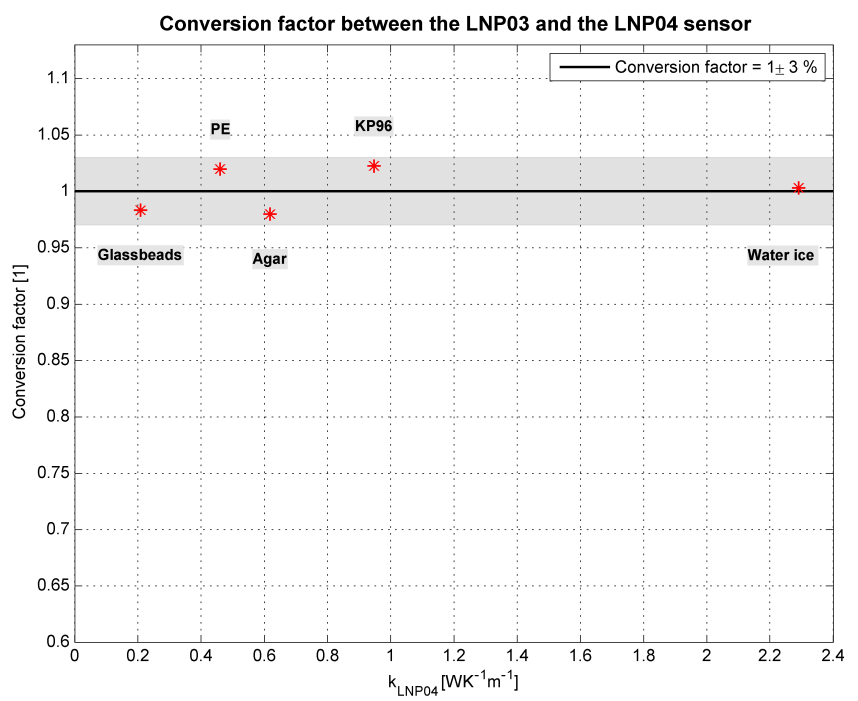

Figure 16. Plot of the conversion factors vs. the thermal conductivities measured by the LNP04 sensor for the different sample materials. The bold line indicates a conversion factor of 1 . Around this line a $\pm 3 \%$ interval shows the scattering area of the factors for the different materials.

dotted lines are a linear fit through these measured values, respectively.

\section{Comparison between the LNP03 and LNP04 sensors}

In this section potential differences in thermal conductivity values, measured by the LNP03 and the LNP04, are shown. The average values of the evaluated conductivities, measured 
in the various sample materials, are listed in Table 10. In Fig. 15 these mean conductivities obtained from the two different sensors, which are represented by the symbols, were plotted against each other, while the dotted line is a linear fit through the data points. From the mean values a conversion factor between the LNP03 and LNP04 sensors was calculated, shown in Table 12. So the following relation can be formulated:

$k_{\mathrm{LNP} 03}=f_{\text {con }} \cdot k_{\mathrm{LNP} 04}$.

These conversion factors vs. the thermal conductivities, measured by the LNP04 sensor, are plotted in Fig. 16. A factor of 1 is indicated by a bold line with a $\pm 3 \%$ interval (shaded area). All conversion factors lie within this range. No trend of the factors is apparent for the different sample materials. Thus, it can be assumed that the difference in length between the LNP03 and LNP04 sensors does not have a significant effect on the determination of the thermal conductivity (as long as the heating periods are not too long).

\section{Conclusions}

In the course of this work various investigations concerning thermal conductivity measurements with two different types of ruggedized, mathematically non-ideal cylindrical sensors were conducted. In a first step the thermal conductivity of different samples was determined by the LNP03 as well as by the shorter LNP04 thermal conductivity probe. Due to the mathematically non-ideal geometry of the LNP03 and the LNP04, it was necessary to calibrate them. The LNP03 sensor has already been calibrated by Kämle et al. (2013), but it was done again with an improved measurement set-up, while the LNP04 was calibrated for the first time. The offthe-shelf TP02 thermal conductivity sensor served as a reference. Average calibration factors $f_{\text {cal }}=0.908$ for the LNP03 and $f_{\text {cal }}=0.909$ for the LNP04 were found. This is closer to 1 (the reference sensor TP02) as found by Kämle et al. (2013) for the LNP03, which was $f_{\text {cal }}=0.80$. The difference between those values can be explained by the adapted measurement set-up, especially the usage of a more accurate data logger for the latest measurements. The main focus was placed on the influence of the probe geometry of non-ideal sensors on measurement results. Thus, the conductivities measured by the LNP03 and LNP04 probes were compared to each other. By performing measurements with both sensors in various sample materials, a conversion factor could be calculated for each sample. The average conversion factor is $f_{\text {con }}=1.002$ and the individual conversion factors lie within a $\pm 3 \%$ range. These results show that there is no significant difference between the conductivities determined by the different LNP probes and confirm that the probe geometry of the rugged non-ideal prototype sensors does not have a significant influence on thermal conductivity values.
It can be noticed that both the measurement uncertainties and the variance are larger for TP02 data than for the data determined by the LNP sensors. This can be explained by having a look at the calculation of the measurement error by Eq. (33), which includes the influence of the resistance of the heating wire, the heating current error and the temperature error. Since the TP02 is a thin and long sensor, much less heating current is needed to provide proper temperature values for the evaluation as for the LNP sensors, which leads to a larger relative heating current error. Additionally the temperature increase in the TP02 while heating is much less than in the LNP sensors, thereby causing a larger relative temperature error. However, the TP02 performs better with regard to certain systematic errors (bias) which have not been considered in our model:

- heat drain through measurement leads; and

- non-ideal geometry (especially finite probe radius and finite probe length).

The smaller temperature increase in TP02 ensures that the heat drain through the measurement leads is mostly negligible for this sensor, in contrast to the LNP. Furthermore, since the geometry of the TP02 almost represents a perfect line heat source, it is better described by the used theoretical model than the LNP03 and the LNP04. Thus, in spite of its smaller precision, the TP02 is a reference in this context due to the reduction of the mentioned systematic errors (except for applications to coarse-grained granular media). This assessment was verified by the described measurements. 


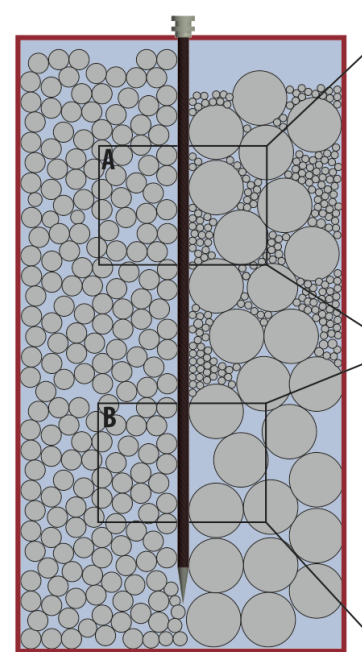

(2014) created by Schaffer A.

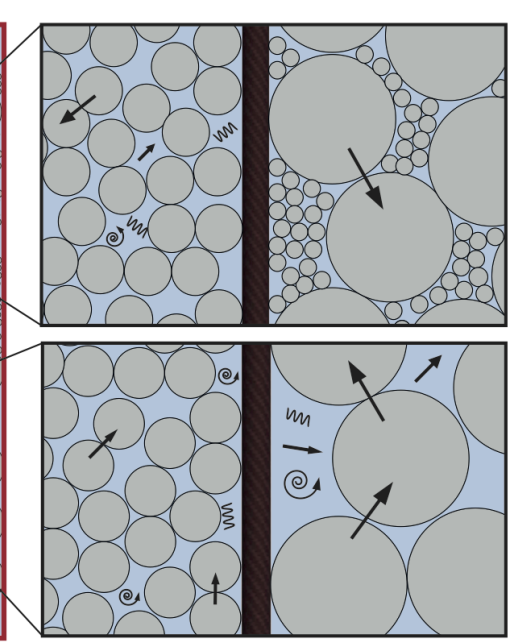

(e) Convection m Radiation $\rightarrow$ Thermal Conduction

Figure A1. Modes of heat transfer in granular media. In the left picture a thermal conductivity probe, inserted into a medium with different particle sizes, can be seen. On the right side of the probe detail A shows the heat transfer processes in a particulate system with differently sized particles, whereas in detail B the heat transfer processes in a system with equally sized particles are shown. The light blue areas represent the voids (Schaffer, 2014).

\section{Appendix A: Heat transfer in granular media}

In this section systems consisting of grainy solid material with small voids in between are discussed. Regolith, a granular and porous material including dust, soil and broken rock, is such a system. Planetary bodies, like the Moon, Mars, asteroids and comets, which are of interest for in situ thermal conductivity measurements and therefore for this work, are covered by layers of regolith. In space the voids between the particles are empty due to the vacuum conditions, whereas under atmospheric pressure these voids are filled with gas.

Particulate systems are characterized by so-called effective properties, which are determined by averaging the properties of the single components (solid and fluid) over the volume. The effective thermal conductivity, $k_{\text {eff }}$, comprises all modes of heat transfer (conduction, radiation and convection). It is the value that can be measured directly by thermal conductivity probes. The effective thermal conductivity is a function of the contributing individual conductivities (Hütter, 2011): $k_{\text {eff }}=f\left(k_{\mathrm{s}}, k_{\mathrm{g}}, k_{\mathrm{sg}}, k_{\mathrm{sc}}, k_{\mathrm{rad}}, k_{\mathrm{conv}}\right)$,

where $k_{\mathrm{s}}$ and $k_{\mathrm{g}}$ are the solid-phase and gas-phase conductivities, respectively, and $k_{\mathrm{sg}}$ describes the heat transport between the solid and gas phases and $k_{\mathrm{sc}}$ the heat transfer between the particles. The radiative heat transfer is expressed as $k_{\text {rad }}$ and the convective heat transfer in the voids as $k_{\text {conv }}$. The evaluation of the respective contribution of the single conductivities is pretty complex and is not further discussed here. Another effective property of such a system is the effective specific heat capacity, which can be calculated by the following formula:

$c_{\mathrm{p}}^{\mathrm{eff}}=\psi \cdot c_{\mathrm{p}}^{\mathrm{void}}+(1-\psi) \cdot c_{\mathrm{p}}^{\mathrm{solid}}$,

where $c_{\mathrm{p}}^{\text {void }}$ and $c_{\mathrm{p}}^{\text {solid }}$ are the specific heat capacities of the voids between the particles and of the solid medium, respectively. The parameter $\psi$ is called porosity and is defined as the ratio of the void-space volume and the total volume of the material:

$\psi=\frac{V_{\text {void }}}{V_{\text {total }}}=\frac{V_{\text {void }}}{V_{\text {solid }}+V_{\text {void }}}$.

A relation between porosity and density can be given as follows:

$\psi=1-\frac{\rho_{\text {bulk }}}{\rho_{\text {solid }}}$,

where $\rho_{\text {bulk }}$ is the bulk density of the total granular medium and $\rho_{\text {solid }}$ is the density of the solid phase alone. In a medium that contains particles of different grain sizes, the porosity is always smaller than in a medium with equally sized particles, because denser packing is possible (see Fig. A1, detail A). 


\section{The Supplement related to this article is available online at doi:10.5194/gi-5-383-2016-supplement.}

Edited by: M. Paton

Reviewed by: three anonymous referees

\section{References}

Abramowitz, M. and Stegun, I. A.: Handbook of mathematical functions with formulas, graphs and mathematical tables, US Department of Commerce, National Bureau of Standards, 1964.

Agilent: Agilent 34970A/34972A data acquisition/switch unit users's guide, Agilent Technologies, 2012.

Blackwell, J.: Transient heat flow problems in cylindrical symmetry, Ph.D. thesis, University of Western Ontario, London, Canada, 1952.

Blackwell, J.: A transient-flow method for determination of thermal constants of insulating materials in bulk, Part 1 - Theory, J. Appl. Phys., 25, 137-144, doi:10.1063/1.1721592, 1954.

Carslaw, H. S. and Jaeger, J. C.: Conduction of heat in solids, 2nd Edn., Oxford University Press, 510 pp., 1959.

ESA: Luna Mission Speed Dating, http://esaconferencebureau.com/ 2014-events/14c05a/ (last access: 5 September 2016), 2014.

Faigle: Data sheet, Material: PAS-PE3, Faigle Kunststoffe $\mathrm{GmbH}$, 2 pp., 2014.

Goodhew, S. and Griffiths, R.: Analysis of thermal-probe measurements using an iterative method to give sample conductivity and diffusivity data, Appl. Energy, 77, 205-223, doi:10.1016/S03062619(03)00122-3, 2004.

Heusler: Datenblatt ISOTAN, Isabellenhütte Heusler GmbH \& Co. KG, 4 pp., 2014.

Hofmeister, A. M., Branlund, J. M., and Pertermann, M.: Treatise on Geophysics, Elsevier, 543-578, 2007.

Hütter, E.: Development and testing of thermal sensors for planetary applications, Ph.D. thesis, University of Graz, 2011.

Hütter, E. S. and Kömle, N. I.: Performance of thermal conductivity probes for planetary applications, Geosci. Instrum. Method. Data Syst., 1, 53-75, doi:10.5194/gi-1-53-2012, 2012.

Hukseflux: TP08 - Small size non-steady-state probe for thermal conductivity measurement, User manual, Hukseflux Thermal Sensors, 43 pp., 2007.

Hukseflux: TP02 - Non-steady-state probe for thermal conductivity measurement, User manual, Hukseflux Thermal Sensors, 2 pp., 2010.

Hukseflux: LNP04 - Construction and functional tests v1304, Hukseflux Thermal Sensors, 13 pp., 2013.

Incropera, F. P., DeWitt, D. P., Bergman, T. L., and Lavine, A. S.: Fundamentals of heat and mass transfer, 6th Edn., John Wiley \& Sons, 1070 pp., 2007.
Jaeger, J.: Conduction of heat in an infinite region bounded internally by a circular cylinder of a perfect conductor, Aust. J. Phys., 9, 167-179, doi:10.1071/PH560167, 1956.

Jones, B.: Thermal conductivity probe: Development of method and application to a coarse granular medium, J. Phys., 21, 832-839, doi:10.1088/0022-3735/21/9/002, 1988.

Kerafol: Keratherm - Thermal grease, Kerafol Keramische Folien GmbH, 1 pp., 2013.

Kämle, N., Macher, W., Kargl, G., and Bentley, M.: Calibration of non-ideal thermal conductivity sensors, Geosci. Instrum. Method. Data Syst., 2, 151-156, doi:10.5194/gi-2-151-2013, 2013.

Kömle, N. I.: Cometary surface processes: Experiments and theory, Solar-Planetary Relations, Research Signpost, 227-259, 2005.

Langseth, M. G. J., Clark, S. P. J., Chute, J. L. J., Kheim, S. J. J., and Wechsler, A. E.: Apollo 15: Preliminary science report (NASA SP-289), chap. Heat flow experiment, National Aeronautics and Space Administration (NASA), 1972.

Langseth, M. G. J., Kheim, S. J. J., and Chute, J. L. J.: Apollo 17: Preliminary science report (NASA SP-330), chap. Heat flow experiment, National Aeronautics and Space Administration (NASA), 1973.

Macher, W., Kämle, N., Bentley, M., and Kargl, G.: The heated infinite cylinder with sheath and two thermal surface resistance layers, Int. J. Heat Mass Transf., 57, 528-534, doi:10.1016/j.ijheatmasstransfer.2012.10.070, 2013.

McGee, T. D.: Principles and methods of temperature measurement, John Wiley \& Sons, USA, 608 pp., 1988.

Özisik, M. N.: Boundary value problems of heat conduction, Dover Phoenix Editions, 504 pp., 1989.

Schaffer, A.: Calibration strategy for a thermal probe applicable in planetary research, Master's thesis, University of Graz, 2014.

Spohn, T., Knollenberg, J., Ball, A., Banaszkiewicz, M., Benkhoff, J., Grott, M., Grygorczuk, J., Hüttig, C., Hagermann, A., Kargl, G., Kaufmann, E., Kämle, N., Kührt, E., Kossacki, K., Marczewski, W., Pelivan, I., Schrädter, R., and Seiferlin, K.: Thermal and mechanical properties of the near-surface layers of comet 67P/Churyumov-Gerasimenko, Science, 349, 6247, doi:10.1126/science.aab0464, 2015.

TTi: TTi instruction manual, Thurlby Thandar Instruments Ltd., 180 pp., 2003.

Wikipedia: http://de.wikipedia.org/wiki/Wärmeleitfähigkeit (last access: 5 September 2016), 2014.

Zent, A., Hecht, M., Cobos, D., Wood, S., Hudson, T., Milkovich, S., DeFlores, L., and Mellon, M.: Initial results from the thermal and electrical conductivity probe (TECP) on Phoenix, J. Geophys. Res., 115., E00E14, doi:10.1029/2009JE003420, 2010. 